\title{
Variation for Agro-Morphological Traits among Kabuli Chickpea (Cicer arietinum L.) Genotypes
}

\author{
S. M. Tesfamichael ${ }^{1}$, S. M. Githiri ${ }^{1}$, A. B. Nyende $^{1} \&$ N. V. P. R. G. Rao \\ ${ }^{1}$ Jomo Kenyatta University of Agriculture and Technology, Nairobi, Kenya \\ ${ }^{2}$ International Crops Research Institute for the Semi - Arid Tropics (ICRISAT), Nairobi, Kenya \\ Correspondence: S. M. Tesfamichael, Jomo Kenyatta University of Agriculture and Technology, Nairobi, Kenya. \\ Tel: 254-71-809-2404. E-mail: tesfamallu@gmail.com
}

\author{
Received: February 24, 2015 Accepted: May 5, 2015 Online Published: June 15, 2015 \\ doi:10.5539/jas.v7n7p75 URL: http://dx.doi.org/10.5539/jas.v7n7p75
}

\begin{abstract}
The objectives of this study were to evaluate genetic variation among kabuli chickpea genotypes and to determine the relationships among agronomic traits with seed yield. Field experiments were conducted during the long and short rain seasons of 2013 using alpha lattice design in triplicate. Data on agro-morphological traits were recorded using descriptors for chickpea and analysed using SAS 2013 and Genstat 2014. There were highly significant $(p<0.001)$ variations among genotypes and genotype by environment interactions for all studied traits. The top five high yielding genotypes were ICCV 05315, ICC 13461, ICCV 07313, ICC 13764 and ICCV 00302. Genotypes ranking for most agronomic traits varied across environments which indicated a crossover type of genotype by environment interactions. Evaluated genotypes were polymorphic for six qualitative traits. Seed yield ha ${ }^{-1}$ was positively and significantly $(\mathrm{p}<0.05)$ correlated with biomass yield ha ${ }^{-1}$, pods plant ${ }^{-1}$, plant canopy width and secondary branches plant ${ }^{-1}$. These characters could be used for indirect selection of high yielding genotypes. The first principal component explained $57 \%$ of the total variation and was associated with days to $50 \%$ flowering and podding, plant canopy width, plant height, number of primary and secondary branches plant ${ }^{-1}$, days to $75 \%$ maturity, number of pods plant ${ }^{-1}$ and biomass yield ha ${ }^{-1}$ as positive contributors. The documented information on genetic variation and association of agronomic traits with seed yield can be exploited to devise suitable breeding strategies and chickpea germplasm conservation.
\end{abstract}

Keywords: Cicer arietinum, correlation, genetic variation, indirect selection

\section{Introduction}

Chickpea (Cicer arietinum L.) is the third most important pulse crop in the world, after dry bean and field pea (Jagadish \& Jayalakshmi, 2014). The crop accounts for 12\% of the world pulses production (Khan et al., 2011). Chickpea is used as human food and livestock feed due to its high protein, vitamins, minerals and fibre contents. It is grown in arid and semi-arid regions of the world with a total annual production of 11.6 million tons from 13.2 million hectares and an average yield of 0.96 tons ha ${ }^{-1}$ (FAOSTAT, 2012). Two main types of cultivated chickpea are grown globally: the desi and kabuli types. The major bottlenecks limiting chickpea production globally include narrow genetic diversity of cultivated chickpea, biotic (Pod borer, Fusarium wilt, and Ascochyta blight) and abiotic (drought, heat, cold and salinity) (Millan et al., 2006, Upadhyaya et al., 2008) stresses.

Chickpea is a relatively new pulse crop in Kenya with a potential yield production of 1.4-2.5 ton ha ${ }^{-1}$ (Onyari et al., 2010), 0.2-2.9 ton $\mathrm{ha}^{-1}$ (Mulwa et al., 2010). The presence of information on genetic variation and interrelationship among yield and yield related traits is a pre-requisite for further breeding programme aimed at improving yield or quality of chickpea. Assessment of available chickpea germplasm can provide practical information for selection of elite plant material and thus, assist in devising future breeding strategies.

Studies in chickpea have reported a significant genetic variation for number of secondary branches plant ${ }^{-1}$, number of pods plant ${ }^{-1}$, biomass yield, seed yield and harvest index (Malik et al., 2009), days to flowering, days to maturity, number of pods plant ${ }^{-1}$ and seed yield (Bakhsh et al., 2003). Other studies in chickpea reported significant variation for growth habit, seed shape and seed coat colour (Qureshi et al., 2004), plant height, number of primary and secondary branches plant ${ }^{-1}$, pods plant ${ }^{-1}$ and biomass yield (Aslamshad et al., 2009).

Previous studies in chickpea have reported positive and significant correlation of seed yield with number of 
secondary branches plant ${ }^{-1}$, plant height; number of pods plant ${ }^{-1}$ and biomass yield but negatively significant correlated with days to flowering, days to maturity and 100-seed weight (Ali \& Ahsan, 2012, Malik et al., 2010). According to Malik et al. (2010) traits with positive and significant correlation with seed yield can be used for indirect selection of high yielding genotypes and used in future chickpea breeding programmes.

Multivariate analysis is a useful statistical method which shows the relationships among essential traits. Principal component analysis is one of the multivariate analyses and it can assist chickpea breeders to determine which characters have major contribution to the total variation. Characterization of available germplasm for important characters will facilitate efficient utilization in chickpea breeding. The objectives of this study were to evaluate the genetic variation and analyze the association among agronomic traits with seed yield.

\section{Materials and Methods}

\subsection{Experimental Sites}

Field experiments were carried out at the University farm of Jomo Kenyatta University of Agriculture and Technology, Juja and at the field station of the University of Nairobi, Kabete, Kenya during the long and short rain seasons of each site. Juja is located about $36 \mathrm{~km}$ North East of Nairobi at $1^{\circ} 11^{\prime} 0^{\prime \prime} \mathrm{S}, 37^{\circ} 7^{\prime} 0^{\prime \prime} \mathrm{E}$ with an altitude of $1530 \mathrm{~m}$ above sea level. It is in upper midland zone 4, semi-humid to semi-arid. It receives an annual rainfall of $600-800 \mathrm{~mm}$ and mean annual temperature of $18.9^{\circ} \mathrm{C}$ and clay soils (Kaluli et al., 2011). Kabete is located about $15 \mathrm{~km}$ to the West of Nairobi and lies at a latitude of $1^{\circ} 15^{\prime} \mathrm{S}$, longitude $36^{\circ} 41^{\prime} \mathrm{E}$ with an altitude of $1940 \mathrm{~m}$ above sea level and is in upper midland zone 3 (Sombroek et al., 1982). It is semi-humid with mean annual rainfall of $1000 \mathrm{~mm}$ and mean annual temperature of $18^{\circ} \mathrm{C}$ and deep and friable reddish or brown, friable clay soils (Karuku et al., 2012). Both experimental sites have bimodal rainfall pattern with peaks in April (long rain season) and November (short rain season).

\subsection{Plant Materials and Experimental Details}

Thirty seven (Table 1) kabuli chickpea genotypes including two check (early maturing, high yielding, resistant to Fusarium wilt) varieties were used in this study. The genotypes were obtained from the International Crops Research Institute for Semi-arid Tropics (ICRISAT) gene bank, Nairobi, Kenya. Prior to planting, the experimental fields were ploughed and manually leveled with the help of spades and forked jembes.

Genotypes were evaluated under field conditions using alpha lattice design with three replications. Each experimental unit consisted of two rows with a gross area of $2.5 \mathrm{~m}^{2}$. Genotypes were randomly allocated to the entire experimental plots in each block within replication at both sites and seasons. Each of the genotypes was sown by hand drilling in two rows with inter row spacing's of $50 \mathrm{~cm}$.

Seeds were sown on $14^{\text {th }}$ May (long rain Kabete), on $28^{\text {th }}$ May (long rain Juja), on $18^{\text {th }}$ November (short rain Kabete) and on $29^{\text {th }}$ December (short rain Juja), 2013 for season one and season two respectively. Two weeks after emergence and establishment, plants were thinned to maintain intra-row spacing's of $10 \mathrm{~cm}$.

The field experiment was rain-fed and supplementary irrigation was provided when necessary. All the cultural practices were performed as recommended for chickpea production. 
Table 1. Kabuli chickpea genotypes used in this study and their country of origin

\begin{tabular}{|c|c|c|c|c|c|}
\hline Entry & Name & Origin & Entry & Name & Origin \\
\hline 1 & ICC 10885 & Ethiopia & 21 & ICCV 08302 & ICRISAT, India \\
\hline 2 & ICC 13283 & Iran & 22 & ICCV 08303 & ICRISAT, India \\
\hline 3 & ICC 13357 & Iran & 23 & ICCV 08307 & ICRISAT, India \\
\hline 4 & ICC 13461 & Iran & 24 & ICCV 92311 & ICRISAT, India \\
\hline 5 & ICC 13764 & Iran & 25 & ICCV 08310 & ICRISAT, India \\
\hline 6 & ICC 15802 & Syria & 26 & ICCV 92318 (Check) & ICRISAT, Kenya \\
\hline 7 & ICC 16654 & China & 27 & ICCV 95311 & ICRISAT, India \\
\hline 8 & ICC 2482 & Iran & 28 & IG 71055 & ICRISAT, India \\
\hline 9 & ICC 7315 & Iran & 29 & ICCV 07304 & ICRISAT, India \\
\hline 10 & ICCV 00302 & ICRISAT, India & 30 & IG 72109 & ICRISAT, India \\
\hline 11 & ICCV 00305 (Check) & ICRISAT, Kenya & 31 & K017 & ICRISAT, India \\
\hline 12 & ICCV 00402 & ICRISAT, India & 32 & K004 & ICRISAT, India \\
\hline 13 & ICCV 03309 & ICRISAT, India & 33 & K012 & ICRISAT, India \\
\hline 14 & ICCV 03404 & ICRISAT, India & 34 & K022 & ICRISAT, India \\
\hline 15 & ICCV 05312 & ICRISAT, India & 35 & K025 & ICRISAT, India \\
\hline 16 & ICCV 05315 & ICRISAT, India & 36 & ICCV 08313 & ICRISAT, India \\
\hline 17 & ICCV 07306 & ICRISAT, India & 37 & K038 & ICRISAT, India \\
\hline 18 & ICCV 06304 & ICRISAT, India & 38 & K041 & ICRISAT, India \\
\hline 19 & ICCV 07308 & ICRISAT, India & 39 & K034 & ICRISAT, India \\
\hline 20 & ICCV 07313 & ICRISAT, India & & & \\
\hline
\end{tabular}

\subsection{Data Collection and Analysis}

Data were recorded based on the available descriptors for chickpea (Cicer arietinum L.) (IBPGR, ICRISAT and ICARDA, 1993). Both qualitative and quantitative data were collected using the provided descriptors. Data such as flower colour, stem colour, growth habit, pod dehiscence and leaf type were scored on plot $^{-1}$ basis while seed coat colour, seed shape and seed testa texture were scored visually on seed samples. Data on days to $50 \%$ flowering, days to $50 \%$ podding, days to $75 \%$ maturity, pod filling period, biomass yield ha ${ }^{-1}$ and seed yield $\mathrm{ha}^{-1}$ were recorded on plot $^{-1}$ basis but the other characters were recorded on six randomly selected and pre-tagged plants from the middle two rows of each plot.

Data were analysed using SAS, Second Edition (SAS Institute Inc., 2013) and Gen-Stat (Gen-Stat release 15.1, 2014) at 5\% level of significance. Correlation analyses among the mean values of quantitative traits were conducted as outlined by Kwon and Torrie (1964).

The mean of each character was standardized prior to principal components analysis in order to avoid differences in measuring scales. Principal component analysis of the standardized mean was performed to investigate the importance of different traits in explaining multivariate variation (Mallikarjuna et al., 2003) based on variance-covariance matrix.

\section{Results}

\subsection{Variation in Agronomic and Yield Traits}

\subsubsection{Day to $50 \%$ Flowering (DF)}

The genotypes and genotype by environment interactions showed highly significant $(p<0.0004)$ variation in days to $50 \%$ flowering (Table 2). All genotypes took longer time to flower in the long rain season compared to the short rain season at Juja. While at Kabete $71.8 \%$ of genotypes took longer time in the short rain compared to the long rain season. Early (ICCV 08302, ICCV 08303 and ICCV 07308) and late (ICC 13283, ICC 13461 and IG 72109) flowering genotypes at Juja consistently flowered early and late at Kabete. Twenty eight and twelve 
genotypes flowered earlier than the check varieties ICCV 00305 and ICCV 92318 respectively.

On the basis of overall means, the studied genotypes showed wide differences in response to flowering time. Eleven genotypes were classified as early (<40 days), 19 genotypes as moderate (40-50 days) and 9 genotypes were late ( $>50$ days) in flowering. The earliest flowering genotypes were ICCV 07308 (31.9 days), ICCV 08303 (32.0 days) and ICCV 08302 (32.5 days) followed by ICCV 03404 (35.6 days) and ICCV 05312 (35.7 days). Genotypes ICC 13283 (64.8 days), ICC 13461 (62.6 days), IG 72109 (58.8 days), ICC 15802 (57.3 days) and K022 (57.3 days) were the latest in flowering (Table 2). The check varieties ICCV 00305 (47.7 days) and ICCV 92318 (40.2 days) were categorized as moderate in flowering. Based on the environment wise means, the earliest flowering was recorded in the short rain season at Juja (37.7 days) followed by long rain season at Juja (46.6 days), long rain season at Kabete (47.6 days) and short rain season at Kabete (48.3 days) with a grand mean of 45.1 days.

\subsubsection{Plant Height $(\mathrm{PH})$}

There were highly significant $(\mathrm{p}<0.0001)$ variations among the genotypes and genotype by environment interactions for plant height (Table 2). All genotypes recorded taller plant height in Juja compared to Kabete. The studied genotypes recorded taller plant height during the long rain season compared to the short rain season at Juja. While at Kabete some genotypes recorded taller plant height during the short rain season and vice versa. The taller (IG 72019, IG 71055 and ICC 13461) and shorter (ICCV 07306, ICCV 92318 and K022) genotypes for plant height at Juja also recorded taller and shorter plant height at Kabete. The ranking of genotypes for plant height varied across environments. Twenty six and thirty six genotypes were taller than the check varieties ICCV 00305 and ICCV 92318 respectively.

Based on overall means, genotypes showed significant genetic variation with respect to plant height. Three genotypes were classified as short $(<40 \mathrm{~cm}), 30$ genotypes as medium $(40.1-50.0 \mathrm{~cm})$ and 6 genotypes had tall ( $>50.0 \mathrm{~cm}$ ) (Table 2) plant height. Genotypes IG $71055(58.9 \mathrm{~cm})$, IG $72109(54.4 \mathrm{~cm})$ ICC $13461(53.8 \mathrm{~cm})$, ICCV $03404(53.0 \mathrm{~cm})$ and K025 $(52.0 \mathrm{~cm})$ recorded the tallest plant height. While genotypes ICCV $07306(37.2$ $\mathrm{cm}), \mathrm{K} 022(39.8 \mathrm{~cm})$, ICCV $92318(39.9 \mathrm{~cm})$ and K017 $(40.3 \mathrm{~cm})$ were the shortest for plant height. The check varieties ICCV $00305(45.6 \mathrm{~cm})$ and ICCV $92318(39.9 \mathrm{~cm})$ were classified as medium and short plant height respectively. With respect to environment wise means, the tallest plant height was recorded in the long rain season at Juja $(56.3 \mathrm{~cm})$ followed by short rain season at Juja $(50.0 \mathrm{~cm})$, short rain season at Kabete $(39.9 \mathrm{~cm})$ and long rain season at Kabete $(39.1 \mathrm{~cm})$ with a grand mean of $46.3 \mathrm{~cm}$.

Table 2. Variation among Kabuli genotypes for days to 50\% flowering and plant height at Juja and Kabete in 2013

\begin{tabular}{|c|c|c|c|c|c|c|c|c|c|c|c|}
\hline \multirow{4}{*}{ Genotypes } & \multicolumn{4}{|c|}{ Days to $50 \%$ Flowering (DF) } & \multirow{4}{*}{$\begin{array}{l}\text { Overall } \\
\text { Mean }\end{array}$} & \multicolumn{4}{|c|}{ Plant Height $(\mathrm{PH})$ in centimeters } & \multirow{4}{*}{$\begin{array}{l}\text { Overall } \\
\text { Mean }\end{array}$} & \multirow{4}{*}{$\begin{array}{l}\text { P. } \\
\text { value }\end{array}$} \\
\hline & Long Rain & Short & Long Rain & Short Rain & & \multirow{3}{*}{$\begin{array}{l}\text { Long Rain } \\
\text { Juja } \\
\text { Mean } \pm \text { S.E }\end{array}$} & \multirow{3}{*}{$\begin{array}{l}\text { Short } \\
\text { Rain Juja } \\
\text { Mean } \pm \text { S.E }\end{array}$} & \multirow{3}{*}{$\begin{array}{l}\text { Long Rain } \\
\text { Kabete } \\
\text { Mean } \pm \text { S.E }\end{array}$} & \multirow{3}{*}{$\begin{array}{l}\text { Short Rain } \\
\text { Kabete } \\
\text { Mean } \pm \text { S.E }\end{array}$} & & \\
\hline & Juja & Rain Juja & Kabete & Kabete & & & & & & & \\
\hline & Mean \pm S.E & Mean \pm S.E & Mean \pm S.E & Mean \pm S.E & & & & & & & \\
\hline ICCV 05315 & $38.0 \pm 3.6$ & $30.9 \pm 1.8$ & $44.5 \pm 4.1$ & $48.3 \pm 1.9$ & 40.4 & $53.4 \pm 3.6$ & $49.4 \pm 2.1$ & $35.6 \pm 3.1$ & $39.7 \pm 1.1$ & 44.5 & $* *$ \\
\hline ICC 13461 & $69.7 \pm 3.6$ & $57.7 \pm 1.82$ & $66.2 \pm 4.1$ & $56.9 \pm 1.9$ & 62.6 & $64.6 \pm 3.7$ & $52.4 \pm 2.1$ & $51.0 \pm 3.1$ & $47.4 \pm 1.1$ & 53.8 & $* *$ \\
\hline ICCV 07313 & $38.1 \pm 3.6$ & $28.1 \pm 1.8$ & $37.5 \pm 4.1$ & $39.4 \pm 1.9$ & 35.8 & $53.4 \pm 3.6$ & $46.9 \pm 2.1$ & $37.5 \pm 3.1$ & $38.4 \pm 1.1$ & 44.1 & $* *$ \\
\hline ICC 13764 & $58.5 \pm 3.6$ & $50.3 \pm 1.8$ & $54.8 \pm 4.1$ & $50.1 \pm 1.9$ & 53.4 & $59.8 \pm 3.7$ & $55.9 \pm 2.1$ & $41.9 \pm 3.1$ & $39.0 \pm 1.1$ & 49.2 & $* *$ \\
\hline ICCV 00302 & $56.3 \pm 3.6$ & $42.5 \pm 12.1$ & $47.1 \pm 4.1$ & $47.6 \pm 1.9$ & 48.4 & $60.8 \pm 3.6$ & $48.24 \pm 2.1$ & $42.0 \pm 3.1$ & $34.6 \pm 1.1$ & 46.4 & $* *$ \\
\hline ICC 2482 & $56.8 \pm 3.6$ & $56.9 \pm 1.8$ & $57.0 \pm 4.7$ & $51.8 \pm 1.9$ & 55.6 & $61.6 \pm 3.6$ & $48.9 \pm 2.1$ & $46.0 \pm 3.5$ & $42.9 \pm 1.1$ & 49.8 & $* *$ \\
\hline ICCV 03404 & $33.6 \pm 3.6$ & $25.3 \pm 1.8$ & $37.9 \pm 4.1$ & $45.5 \pm 1.9$ & 35.6 & $63.1 \pm 3.6$ & $57.1 \pm 2.1$ & $50.8 \pm 3.5$ & $41.2 \pm 1.1$ & 53.0 & $* *$ \\
\hline ICCV 00402 & $45.4 \pm 3.6$ & $33.6 \pm 1.8$ & $47.6 \pm 4.1$ & $52.9 \pm 1.9$ & 44.9 & $53.3 \pm 3.6$ & $49.0 \pm 2.1$ & $32.6 \pm 3.1$ & $39.8 \pm 1.1$ & 43.7 & $* *$ \\
\hline ICCV 07306 & $35.2 \pm 3.6$ & $30.0 \pm 1.8$ & $44.8 \pm 4.1$ & $38.6 \pm 1.9$ & 37.2 & $50.1 \pm 3.6$ & $42.1 \pm 2.1$ & $24.2 \pm 3.1$ & $32.5 \pm 1.1$ & 37.2 & $* *$ \\
\hline $\begin{array}{l}\text { ICCV } 00305 \\
\text { (Check) }\end{array}$ & $52 \pm 3.6$ & $39.7 \pm 1.8$ & $48.0 \pm 4.1$ & $51.3 \pm 1.9$ & 47.7 & $59.3 \pm 3.6$ & $47.0 \pm 2.1$ & $41.9 \pm 3.1$ & $34.3 \pm 1.1$ & 45.6 & $* *$ \\
\hline ICCV 05312 & $32.7 \pm 3.6$ & $25.3 \pm 1.8$ & $41.1 \pm 4.1$ & $43.7 \pm 1.9$ & 35.7 & $51.0 \pm 3.6$ & $43.2 \pm 2.1$ & $36.6 \pm 3.1$ & $35.4 \pm 1.1$ & 41.6 & $* *$ \\
\hline IG 71055 & $66.1 \pm 3.6$ & $57.8 \pm 1.8$ & $56.4 \pm 4.1$ & $42.6 \pm 1.9$ & 55.7 & $63.5 \pm 3.6$ & $64.5 \pm 2.1$ & $51.8 \pm 3.1$ & $56.0 \pm 1.1$ & 58.9 & $* *$ \\
\hline ICCV 08302 & $31.9 \pm 3.6$ & $22.6 \pm 1.8$ & $32.1 \pm 4.1$ & $43.5 \pm 1.9$ & 32.5 & $56.0 \pm 3.6$ & $52.1 \pm 2.1$ & $42.5 \pm 3.1$ & $35.7 \pm 1.1$ & 46.6 & $* *$ \\
\hline ICCV 92311 & $37.4 \pm 3.6$ & $27.5 \pm 1.8$ & $42.4 \pm 4.1$ & $53.2 \pm 1.9$ & 40.1 & $54.3 \pm 3.6$ & $48.3 \pm 2.1$ & $39.9 \pm 3.1$ & $33.0 \pm 1.1$ & 43.9 & $* *$ \\
\hline ICC 15802 & $63.3 \pm 3.6$ & $54.5 \pm 1.8$ & $56.8 \pm 4.1$ & $54.4 \pm 1.9$ & 57.3 & $50.4 \pm 3.6$ & $48.4 \pm 2.1$ & $42.6 \pm 3.5$ & $37.1 \pm 1.1$ & 44.6 & $* *$ \\
\hline
\end{tabular}




\begin{tabular}{|c|c|c|c|c|c|c|c|c|c|c|c|}
\hline ICCV 07308 & $29.6 \pm 3.6$ & $23.2 \pm 1.8$ & $32.0 \pm 4.7$ & $42.8 \pm 1.9$ & 31.9 & $54.4 \pm 3.6$ & $52.0 \pm 2.1$ & $34.4 \pm 3.5$ & $39.6 \pm 1.1$ & 45.1 & $* *$ \\
\hline ICC $10885^{\circ}$ & $46.6 \pm 12.1$ & $37.7 \pm 12.1$ & $47.6 \pm 12.2$ & $48.3 \pm 12.1$ & 45.1 & $56.3 \pm 6.7$ & $50.0 \pm 6.6$ & $39.1 \pm 6.6$ & $39.9 \pm 6.6$ & 46.3 & $* *$ \\
\hline ICC $13357^{\circ}$ & $46.6 \pm 12.1$ & $37.7 \pm 12.1$ & $47.6 \pm 12.2$ & $48.3 \pm 12.1$ & 45.1 & $56.3 \pm 6.7$ & $50.0 \pm 6.6$ & $39.1 \pm 6.6$ & $39.9 \pm 6.6$ & 46.3 & $* *$ \\
\hline ICC $16654^{\circ}$ & $46.6 \pm 12.1$ & $37.7 \pm 12.1$ & $47.6 \pm 12.2$ & $48.3 \pm 12.1$ & 45.1 & $56.3 \pm 6.7$ & $50.0 \pm 6.6$ & $39.1 \pm 6.6$ & $39.9 \pm 6.6$ & 46.3 & $* *$ \\
\hline $\operatorname{ICC} 7315^{\circ}$ & $46.6 \pm 12.1$ & $37.7 \pm 12.1$ & $47.6 \pm 12.2$ & $48.3 \pm 12.1$ & 45.1 & $56.3 \pm 6.7$ & $50.0 \pm 6.6$ & $39.1 \pm 6.6$ & $39.9 \pm 6.6$ & 46.3 & $* *$ \\
\hline ICCV $03309^{\circ}$ & $46.6 \pm 12.1$ & $37.7 \pm 12.1$ & $47.6 \pm 12.2$ & $48.3 \pm 12.1$ & 45.1 & $56.3 \pm 6.7$ & $50.0 \pm 6.6$ & $39.1 \pm 6.6$ & $39.9 \pm 6.6$ & 46.3 & $* *$ \\
\hline ICCV $06304^{\circ}$ & $46.6 \pm 12.1$ & $37.7 \pm 12.1$ & $47.6 \pm 12.2$ & $48.3 \pm 12.1$ & 45.1 & $56.3 \pm 6.7$ & $50.0 \pm 6.6$ & $39.1 \pm 6.6$ & $39.9 \pm 6.6$ & 46.3 & $* *$ \\
\hline ICCV $08310^{\circ}$ & $46.6 \pm 12.1$ & $37.7 \pm 12.1$ & $47.6 \pm 12.2$ & $48.3 \pm 12.1$ & 45.1 & $56.3 \pm 6.7$ & $50.0 \pm 6.6$ & $39.1 \pm 6.6$ & $39.9 \pm 6.6$ & 46.3 & $* *$ \\
\hline $\operatorname{ICCV~} 07304^{\circ}$ & $46.6 \pm 12.1$ & $37.7 \pm 12.1$ & $47.6 \pm 12.2$ & $48.3 \pm 12.1$ & 45.1 & $56.3 \pm 6.7$ & $50.0 \pm 6.6$ & $39.1 \pm 6.6$ & $39.9 \pm 6.6$ & 46.3 & $* *$ \\
\hline $\mathrm{K} 004^{\circ}$ & $46.6 \pm 12.1$ & $37.7 \pm 12.1$ & $47.6 \pm 12.2$ & $48.3 \pm 12.1$ & 45.1 & $56.3 \pm 6.7$ & $50.0 \pm 6.6$ & $39.1 \pm 6.6$ & $39.9 \pm 6.6$ & 46.3 & $* *$ \\
\hline $\mathrm{K} 012^{\circ}$ & $46.6 \pm 12.1$ & $37.7 \pm 12.1$ & $47.6 \pm 12.2$ & $48.3 \pm 12.1$ & 45.1 & $56.3 \pm 6.7$ & $50.0 \pm 6.6$ & $39.1 \pm 6.6$ & $39.9 \pm 6.6$ & 46.3 & $* *$ \\
\hline $\operatorname{ICCV~} 08313^{\circ}$ & $46.6 \pm 12.1$ & $37.7 \pm 12.1$ & $47.6 \pm 12.2$ & $48.3 \pm 12.1$ & 45.1 & $56.3 \pm 6.7$ & $50.0 \pm 6.6$ & $39.1 \pm 6.6$ & $39.9 \pm 6.6$ & 46.3 & $* *$ \\
\hline $\mathrm{K} 034^{\circ}$ & $46.6 \pm 12.1$ & $37.7 \pm 12.1$ & $47.6 \pm 12.2$ & $48.3 \pm 12.1$ & 45.1 & $56.3 \pm 6.7$ & $50.0 \pm 6.6$ & $39.1 \pm 6.6$ & $39.9 \pm 6.6$ & 46.3 & $* *$ \\
\hline IG 72109 & $60.5 \pm 3.6$ & $57.7 \pm 1.8$ & $61.3 \pm 4.1$ & $55.7 \pm 1.9$ & 58.8 & $67.3 \pm 3.6$ & $58.9 \pm 2.1$ & $43.2 \pm 3.1$ & $48.1 \pm 1.1$ & 54.4 & $* *$ \\
\hline ICCV 08303 & $33.0 \pm 3.6$ & $22.9 \pm 1.8$ & $34.6 \pm 4.1$ & $37.6 \pm 1.9$ & 32.0 & $54.0 \pm 3.6$ & $48.5 \pm 2.1$ & $28.8 \pm 3.1$ & $35.1 \pm 1.1$ & 41.6 & $* *$ \\
\hline ICCV 95311 & $65.5 \pm 4.3$ & $43.9 \pm 1.8$ & $56 \pm 4.1$ & $58.0 \pm 1.9$ & 55.9 & $56.0 \pm 3.6$ & $52.1 \pm 2.1$ & $37.4 \pm 3.1$ & $38.1 \pm 1.1$ & 45.9 & $* *$ \\
\hline K041 & $38.4 \pm 3.6$ & $29.2 \pm 1.8$ & $36.9 \pm 4.1$ & $47.6 \pm 1.9$ & 38.0 & $53.8 \pm 3.6$ & $48.4 \pm 2.1$ & $42.5 \pm 3.1$ & $36.9 \pm 1.1$ & 45.4 & $* *$ \\
\hline K022 & $65.3 \pm 4.3$ & $55.2 \pm 1.8$ & $58.0 \pm 4.1$ & $50.7 \pm 1.9$ & 57.3 & $51.1 \pm 4.0$ & $41.4 \pm 2.1$ & $31.9 \pm 3.1$ & $34.7 \pm 1.1$ & 39.8 & $* *$ \\
\hline K017 & $44.0 \pm 3.6$ & $31.5 \pm 1.8$ & $49.2 \pm 4.1$ & $40.1 \pm 1.9$ & 41.2 & $52.3 \pm 3.6$ & $46.3 \pm 2.1$ & $24.3 \pm 3.5$ & $38.2 \pm 1.1$ & 40.3 & $* *$ \\
\hline K025 & $32.1 \pm 4.3$ & $24.8 \pm 1.8$ & $42.5 \pm 4.1$ & $50.9 \pm 1.9$ & 37.6 & $60.9 \pm 4.0$ & $52.2 \pm 2.1$ & $44.4 \pm 3.1$ & $50.5 \pm 1.1$ & 52.0 & $* *$ \\
\hline ICCV 08307 & $35.3 \pm 3.6$ & $25.0 \pm 1.8$ & $38.1 \pm 4.1$ & $46.5 \pm 1.9$ & 36.2 & $52.3 \pm 3.6$ & $49.6 \pm 2.1$ & $40.0 \pm 3.1$ & $44.7 \pm 1.1$ & 46.6 & $* *$ \\
\hline $\begin{array}{l}\text { ICCV } 92318 \\
\text { (Check) }\end{array}$ & $32.0 \pm 3.6$ & $34.6 \pm 1.8$ & $43.3 \pm 4.1$ & $50.8 \pm 1.9$ & 40.2 & $50.8 \pm 3.6$ & $43.6 \pm 2.1$ & $31.4 \pm 3.1$ & $33.8 \pm 1.1$ & 39.9 & $* *$ \\
\hline ICC 13283 & $72.3 \pm 4.3$ & $59.0 \pm 1.8$ & $75.1 \pm 4.1$ & $52.7 \pm 1.9$ & 64.8 & $60.7 \pm 4.0$ & $52.0 \pm 2.1$ & $47.5 \pm 3.1$ & $43.1 \pm 1.0$ & 50.8 & $* *$ \\
\hline K038 & $36.5 \pm 3.6$ & $27.4 \pm 1.8$ & $43.6 \pm 4.1$ & $51.1 \pm 1.9$ & 39.6 & $52.9 \pm 3.6$ & $51.6 \pm 2.1$ & $33.3 \pm 3.1$ & $46.5 \pm 1.1$ & 46.1 & $* *$ \\
\hline Mean & 46.6 & 37.7 & 47.6 & 48.3 & 45.1 & 56.3 & 50.0 & 39.1 & 39.9 & 46.3 & \\
\hline Minimum & 72.3 & 59.0 & 75.1 & 58.0 & 64.8 & 50.1 & 41.4 & 24.2 & 32.5 & 37.2 & \\
\hline Maximum & 29.6 & 22.6 & 32.0 & 37.6 & 31.9 & 67.3 & 64.5 & 51.8 & 56.0 & 58.9 & \\
\hline
\end{tabular}

Note. ${ }^{* *}$ - significant at $1 \%,{ }^{*}$ - significant at $5 \%$ and NS - non-significant at $5 \%$ level of significant, ${ }^{\circ}$ - Genotypes were analysed using missing block and hence they have the same values for all traits.

\subsubsection{Days to $75 \%$ Maturity (DM)}

Genotypes and genotype by environment interactions showed highly significant $(p<0.0001)$ variation for days to $75 \%$ physiological maturity (Table 3 ). The evaluated genotypes took longer time to mature in the long rain seasons compared to the short rain seasons at both sites. All genotypes took shorter time to maturity at Juja compared to Kabete in both seasons. The ranking of genotypes for days to maturity varied across environments. Twenty one and six genotypes matured earlier than the check varieties ICCV 00305 and ICCV 92318 individually.

With respect to an overall means, the genotypes showed significant differences in response to maturity time. Ten genotypes were classified as early $(<112$ days), 20 genotypes as moderate (112.0-115.0 days) and 9 genotypes were late ( $>115.0$ days) in maturity. The earliest maturing genotypes were ICCV 08303 (108.8 days), ICCV 07308 (110.0 days), K041 (110.4 days) and ICCV 05312 (110.5 days). While genotypes IG 71055 (123.4 days), ICC 13283 (121.4 days), IG 72109 (120.3 days), ICC 13461 (119.6 days) and ICCV 00302 (118.3 days) were the latest in maturity (Table 3). The check varieties ICCV 00305 (114.4 days) and ICCV 92318 (112.9 days) were classified as moderate in maturity. Based on environment wise means, the earliest maturity was recorded in the short rain season at Juja (91.6 days) followed by short rain season at Kabete (104.7 days), long rain season at Juja (121.6 days) and long rain season at Kabete (139.4 days) with a grand mean of 114.3 days.

\subsubsection{Number of Pods Plant ${ }^{-1}\left(\mathrm{NPP}^{-1}\right)$}

There were highly significant $(\mathrm{p}<0.001)$ differences among genotypes and genotype by environment interactions for number of pods plant ${ }^{-1}$ (Table 3 ). Twenty eight genotypes recorded higher number of pods plant ${ }^{-1}$ 
during the long rain season compared to the short rain season at Juja. While at Kabete, most genotypes except ICC 2482, IG 72109 and K017 recorded higher number of pods plant ${ }^{-1}$ during the long rain season. Many genotypes except ICC 13764, ICC 15802, ICC 2482; ICCV 00302, ICCV 08302 and ICCV 92311 recorded higher number of pods plant ${ }^{-1}$ during the long rain season at Juja compared to long rain season at Kabete. The studied genotypes recorded lower number of pods plant ${ }^{-1}$ during the short rain season at Kabete except ICC 2482 compared to Juja. Genotypes ranking for number of pods plant ${ }^{-1}$ differed across environments. Seven and thirty seven genotypes exceeded the check varieties ICCV 00305 and ICCV 92318 respectively for number of pods plant ${ }^{-1}$ which indicated the existence of genetic diversity.

Based on overall means, the evaluated genotypes showed wide variation with respect to number of pods plant ${ }^{-1}$. Eight genotypes were classified as low (<60), 6 genotypes as moderate (60.0-70.0) and 25 genotypes had high ( $>70$ ) number of pods plant ${ }^{-1}$. Genotype IG 71055 (139.1) followed by ICC 13461 (133.0), ICC 13764 (107.7), ICCV 00302 (100.6) and IG 72109 (89.1) recorded the highest number of pods plant ${ }^{-1}$. Genotypes K038 (37.9), ICCV 92318 (44.2), ICCV 07308 (49.7), ICCV 05312 (51.6) and ICCV 08302 (51.9) recorded the lowest number of pods plant ${ }^{-1}$. The check varieties ICCV 00305 (84.1) and ICCV 92318 (44.2) were classified as high and low pod yielder respectively. With respect to environment-wise means, the highest number of pods plant ${ }^{-1}$ was recorded in the long rain season at Juja (91.5) followed by short rain season at Juja (80.6), long rain season at Kabete (75.4) and short rain season at Kabete (46.8) with a grand mean of 73.6.

Table 3. Variation among Kabuli genotypes for days to $75 \%$ maturity and number of pods plant ${ }^{-1}$ at Juja and Kabete in 2013

\begin{tabular}{|c|c|c|c|c|c|c|c|c|c|c|c|}
\hline \multirow{4}{*}{ Genotypes } & \multicolumn{4}{|c|}{ Days to $75 \%$ Maturity (DM) } & \multirow{4}{*}{$\begin{array}{l}\text { Overall } \\
\text { Mean }\end{array}$} & \multicolumn{4}{|c|}{ Number of Pod Plant ${ }^{-1}\left(\mathrm{NPP}^{-1}\right)$} & \multirow{4}{*}{$\begin{array}{l}\text { Overall } \\
\text { Mean }\end{array}$} & \multirow{4}{*}{$\begin{array}{l}\mathrm{P} . \\
\text { value }\end{array}$} \\
\hline & Long Rain & Short & Long Rain & Short Rain & & \multirow{3}{*}{$\begin{array}{l}\text { Long Rain } \\
\text { Juja } \\
\text { Mean } \pm \text { S.E }\end{array}$} & \multirow{3}{*}{$\begin{array}{l}\text { Short } \\
\text { Rain Juja } \\
\text { Mean } \pm \text { S.E }\end{array}$} & \multirow{3}{*}{$\begin{array}{l}\text { Long Rain } \\
\text { Kabete } \\
\text { Mean } \pm \text { S.E }\end{array}$} & \multirow{3}{*}{$\begin{array}{l}\text { Short Rain } \\
\text { Kabete } \\
\text { Mean } \pm \text { S.E }\end{array}$} & & \\
\hline & Juja & Rain Juja & Kabete & Kabete & & & & & & & \\
\hline & Mean \pm S.E & Mean \pm S.E & Mean \pm S.E & Mean \pm S.E & & & & & & & \\
\hline ICCV 05315 & $118.1 \pm 2.2$ & $92.1 \pm 1.2$ & $138.9 \pm 2.4$ & $98.2 \pm 1.8$ & 111.8 & $74.6 \pm 9.4$ & $107.4 \pm 4.1$ & $58.4 \pm 7.8$ & $52.0 \pm 2.4$ & 73.1 & $* *$ \\
\hline ICC 13461 & $127.1 \pm 2.2$ & $97.2 \pm 1.2$ & $139.2 \pm 2.4$ & $115.0 \pm 1.8$ & 119.6 & $169.1 \pm 9.4$ & $135.0 \pm 4.1$ & $149.3 \pm 7.8$ & $78.4 \pm 2.4$ & 133.0 & $* *$ \\
\hline ICCV 07313 & $123.3 \pm 2.2$ & $86.4 \pm 1.2$ & $136.2 \pm 2.4$ & $103.3 \pm 1.8$ & 112.3 & $147.0 \pm 11.3$ & $89.0 \pm 4.1$ & $67.8 \pm 7.8$ & $36.2 \pm 2.4$ & 85.0 & $* *$ \\
\hline ICC 13764 & $125.7 \pm 2.2$ & $101.0 \pm 1.2$ & $140.0 \pm 2.4$ & $103.9 \pm 1.8$ & 117.6 & $107.3 \pm 9.4$ & $152.5 \pm 4.1$ & $116.8 \pm 7.8$ & $54.0 \pm 2.4$ & 107.7 & $* *$ \\
\hline ICCV 00302 & $127.5 \pm 2.2$ & $101.6 \pm 1.2$ & $138.2 \pm 2.4$ & $105.8 \pm 1.8$ & 118.3 & $77.4 \pm 9.4$ & $146.5 \pm 4.1$ & $118.5 \pm 7.8$ & $60.1 \pm 2.4$ & 100.6 & $* *$ \\
\hline ICC 2482 & $124.5 \pm 2.2$ & $93.7 \pm 1.2$ & $140.2 \pm 2.7$ & $108.5 \pm 1.8$ & 116.7 & $68.9 \pm 11.3$ & $42.9 \pm 4.1$ & $86.4 \pm 9.5$ & $89.6 \pm 2.4$ & 71.9 & $* *$ \\
\hline ICCV 03404 & $119.4 \pm 2.2$ & $91.7 \pm 1.2$ & $137.7 \pm 2.4$ & $109.4 \pm 1.8$ & 114.5 & $95.3 \pm 9.4$ & $66.1 \pm 4.1$ & $42.9 \pm 7.8$ & $38.3 \pm 2.4$ & 60.7 & $* *$ \\
\hline ICCV 00402 & $120.9 \pm 2.2$ & $89.3 \pm 1.2$ & $143.7 \pm 2.4$ & $106.1 \pm 1.8$ & 115.0 & $69.0 \pm 11.3$ & $131.3 \pm 4.1$ & $69.6 \pm 7.8$ & $73.2 \pm 2.4$ & 85.8 & $* *$ \\
\hline ICCV 07306 & $122.4 \pm 2.2$ & $92.0 \pm 1.2$ & $142.5 \pm 2.4$ & $104.9 \pm 1.8$ & 115.4 & $79.8 \pm 9.4$ & $102.7 \pm 4.1$ & $53.2 \pm 7.8$ & $32.2 \pm 2.4$ & 67.0 & $* *$ \\
\hline $\begin{array}{l}\text { ICCV } 00305 \\
\text { (Check) }\end{array}$ & $125.2 \pm 2.2$ & $91.1 \pm 1.2$ & $139.6 \pm 2.4$ & $101.8 \pm 1.8$ & 114.4 & $136.2 \pm 9.4$ & $61.0 \pm 4.1$ & $89.6 \pm 7.8$ & $49.6 \pm 2.4$ & 84.1 & $* *$ \\
\hline ICCV 05312 & $118.4 \pm 2.2$ & $85.0 \pm 1.2$ & $139.7 \pm 2.4$ & $98.8 \pm 1.8$ & 110.5 & $59.3 \pm 9.4$ & $55.6 \pm 4.1$ & $49.4 \pm 7.8$ & $42.1 \pm 2.4$ & 51.6 & $* *$ \\
\hline IG 71055 & $127.3 \pm 2.2$ & $98.1 \pm 1.2$ & $149.2 \pm 2.4$ & $119.0 \pm 1.8$ & 123.4 & $208.1 \pm 9.4$ & $115.0 \pm 4.1$ & $181.6 \pm 7.8$ & $51.8 \pm 2.4$ & 139.1 & $* *$ \\
\hline ICCV 08302 & $119.5 \pm 2.2$ & $88.7 \pm 1.2$ & $139.6 \pm 2.4$ & $96.0 \pm 1.8$ & 110.9 & $62.5 \pm 9.4$ & $76.2 \pm 4.1$ & $65.5 \pm 7.8$ & $22.8 \pm 2.4$ & 56.7 & $* *$ \\
\hline ICCV 92311 & $121.2 \pm 2.2$ & $88.3 \pm 1.2$ & $138.7 \pm 2.4$ & $105.6 \pm 1.8$ & 113.4 & $66.1 \pm 11.3$ & $69.5 \pm 4.1$ & $76.1 \pm 9.5$ & $35.0 \pm 2.4$ & 61.7 & $* *$ \\
\hline ICC 15802 & $119.5 \pm 2.2$ & $91.9 \pm 1.2$ & $141.3 \pm 2.4$ & $103.3 \pm 1.8$ & 114.0 & $54.0 \pm 11.3$ & $115.6 \pm 4.1$ & $103.9 \pm 7.8$ & $39.8 \pm 2.4$ & 78.3 & $* *$ \\
\hline ICCV 07308 & $120.2 \pm 2.2$ & $89.2 \pm 1.2$ & $134.4 \pm 2.7$ & $96.3 \pm 1.8$ & 110.0 & $54.8 \pm 9.4$ & $55.5 \pm 4.1$ & $49.3 \pm 9.5$ & $38.9 \pm 2.4$ & 49.7 & $* *$ \\
\hline ICC $10885^{\circ}$ & $121.6 \pm 5.2$ & $91.6 \pm 5.2$ & $139.4 \pm 5.3$ & $104.7 \pm 5.2$ & 114.3 & $91.5 \pm 34.4$ & $80.6 \pm 34.3$ & $75.4 \pm 34.4$ & $46.8 \pm 34.3$ & 73.6 & $* *$ \\
\hline $\operatorname{ICC} 13357^{\circ}$ & $121.6 \pm 5.2$ & $91.6 \pm 5.2$ & $139.4 \pm 5.3$ & $104.7 \pm 5.2$ & 114.3 & $91.5 \pm 34.4$ & $80.6 \pm 34.3$ & $75.4 \pm 34.4$ & $46.8 \pm 2.4$ & 73.6 & $* *$ \\
\hline ICC $16654^{\circ}$ & $121.6 \pm 5.2$ & $91.6 \pm 5.2$ & $139.4 \pm 5.3$ & $104.7 \pm 5.2$ & 114.3 & $91.5 \pm 34.4$ & $80.6 \pm 34.3$ & $75.4 \pm 34.4$ & $46.8 \pm 34.3$ & 73.6 & $* *$ \\
\hline $\operatorname{ICC} 7315^{\circ}$ & $121.6 \pm 5.2$ & $91.6 \pm 5.2$ & $139.4 \pm 5.3$ & $104.7 \pm 5.2$ & 114.3 & $91.5 \pm 34.4$ & $80.6 \pm 34.3$ & $75.4 \pm 34.4$ & $46.8 \pm 34.4$ & 73.6 & $* *$ \\
\hline ICCV $03309^{\circ}$ & $121.6 \pm 5.2$ & $91.6 \pm 5.2$ & $139.4 \pm 5.3$ & $104.7 \pm 5.2$ & 114.3 & $91.5 \pm 34.4$ & $80.6 \pm 34.3$ & $75.4 \pm 34.4$ & $46.8 \pm 34.4$ & 73.6 & $* *$ \\
\hline
\end{tabular}




\begin{tabular}{|c|c|c|c|c|c|c|c|c|c|c|c|}
\hline ICCV $06304^{\circ}$ & $121.6 \pm 5.2$ & $91.6 \pm 5.2$ & $139.4 \pm 5.3$ & $104.7 \pm 5.2$ & 114.3 & $91.5 \pm 34.4$ & $80.6 \pm 34.3$ & $75.4 \pm 34.4$ & $46.8 \pm 34.3$ & 73.6 & $* *$ \\
\hline ICCV $08310^{\circ}$ & $121.6 \pm 5.2$ & $91.6 \pm 5.2$ & $139.4 \pm 5.3$ & $104.7 \pm 5.2$ & 114.3 & $91.5 \pm 34.4$ & $80.6 \pm 34.3$ & $75.4 \pm 34.4$ & $46.8 \pm 34.3$ & 73.6 & $* *$ \\
\hline ICCV $07304^{\circ}$ & $121.6 \pm 5.2$ & $91.6 \pm 5.2$ & $139.4 \pm 5.3$ & $104.7 \pm 5.2$ & 114.3 & $91.6 \pm 34.4$ & $80.6 \pm 34.3$ & $75.4 \pm 34.4$ & $46.8 \pm 34.3$ & 73.6 & $* *$ \\
\hline $\mathrm{K} 004^{\circ}$ & $121.6 \pm 5.2$ & $91.6 \pm 5.2$ & $139.4 \pm 5.3$ & $104.7 \pm 5.2$ & 114.3 & $91.5 \pm 34.4$ & $80.6 \pm 34.3$ & $75.4 \pm 34.4$ & $46.8 \pm 34.3$ & 73.6 & $* *$ \\
\hline $\mathrm{K} 012^{\circ}$ & $121.6 \pm 5.2$ & $91.6 \pm 5.2$ & $139.4 \pm 5.3$ & $104.7 \pm 5.2$ & 114.3 & $91.5 \pm 34.4$ & $80.6 \pm 34.3$ & $75.4 \pm 34.4$ & $46.8 \pm 34.3$ & 73.6 & $* *$ \\
\hline ICCV $08313^{\circ}$ & $121.6 \pm 5.2$ & $91.6 \pm 5.2$ & $139.4 \pm 5.3$ & $104.7 \pm 5.2$ & 114.3 & $91.5 \pm 34.4$ & $80.6 \pm 34.3$ & $75.4 \pm 34.4$ & $46.8 \pm 34.3$ & 73.6 & $* *$ \\
\hline $\mathrm{K} 034^{\circ}$ & $121.6 \pm 5.2$ & $91.6 \pm 5.2$ & $139.4 \pm 5.3$ & $104.7 \pm 1.8$ & 114.3 & $91.5 \pm 34.4$ & $80.6 \pm 34.3$ & $75.4 \pm 34.4$ & $46.8 \pm 34.3$ & 73.6 & $* *$ \\
\hline IG 72109 & $124.2 \pm 2.2$ & $97.5 \pm 1.2$ & $141.4 \pm 2.4$ & $118.0 \pm 1.8$ & 120.3 & $127.6 \pm 9.4$ & $96.3 \pm 4.1$ & $48.8 \pm 7.8$ & $83.8 \pm 2.4$ & 89.1 & $* *$ \\
\hline ICCV 08303 & $118.7 \pm 2.2$ & $87.0 \pm 1.2$ & $135.5 \pm 2.4$ & $93.8 \pm 1.8$ & 108.7 & $61.7 \pm 9.4$ & $55.4 \pm 4.1$ & $57.6 \pm 7.8$ & $33.1 \pm 2.4$ & 51.9 & $* *$ \\
\hline ICCV 95311 & $124.7 \pm 2.2$ & $90.0 \pm 1.2$ & $140.3 \pm 2.4$ & $110.3 \pm 1.8$ & 116.3 & $92.4 \pm 9.4$ & $73.5 \pm 4.1$ & $92.4 \pm 7.8$ & $40.7 \pm 2.4$ & 74.8 & $* *$ \\
\hline K041 & $117.0 \pm 2.2$ & $89.9 \pm 1.2$ & $135.7 \pm 2.4$ & $99.0 \pm 1.8$ & 110.4 & $62.7 \pm 11.3$ & $65.5 \pm 4.1$ & $85.4 \pm 7.8$ & $40.8 \pm 2.4$ & 63.6 & $* *$ \\
\hline K022 & $123.3 \pm 2.6$ & $94.1 \pm 1.2$ & $139.4 \pm 2.4$ & $100.5 \pm 1.8$ & 114.3 & $121.6 \pm 11.3$ & $44.1 \pm 4.1$ & $59.7 \pm 7.8$ & $38.0 \pm 2.4$ & 65.8 & $* *$ \\
\hline K017 & $119.8 \pm 2.2$ & $87.8 \pm 1.2$ & $136.9 \pm 2.4$ & $101.6 \pm 1.8$ & 111.5 & $84.3 \pm 9.4$ & $70.2 \pm 4.1$ & $50.1 \pm 7.8$ & $74.4 \pm 2.4$ & 69.7 & $* *$ \\
\hline K025 & $114.9 \pm 2.6$ & $86.9 \pm 1.2$ & $140.8 \pm 2.4$ & $103.1 \pm 1.8$ & 111.4 & $68.9 \pm 11.3$ & $59.8 \pm 4.1$ & $46.1 \pm 7.8$ & $38.7 \pm 2.4$ & 53.4 & $* *$ \\
\hline ICCV 08307 & $116.8 \pm 2.2$ & $86.6 \pm 1.2$ & $137.7 \pm 2.4$ & $101.2 \pm 1.8$ & 110.6 & $61.7 \pm 9.4$ & $61.8 \pm 4.1$ & $50.3 \pm 7.8$ & $39.9 \pm 2.4$ & 53.4 & $* *$ \\
\hline $\begin{array}{l}\text { ICCV } 92318 \\
\text { (Check) }\end{array}$ & $117.8 \pm 2.2$ & $92.8 \pm 1.2$ & $137.7 \pm 2.4$ & $103.4 \pm 1.8$ & 112.9 & $66.9 \pm 9.4$ & $42.7 \pm 4.1$ & $35.9 \pm 7.8$ & $31.3 \pm 2.4$ & 44.2 & $* *$ \\
\hline ICC 13283 & $126.1 \pm 2.6$ & $98.2 \pm 1.2$ & $142.3 \pm 2.4$ & $119.0 \pm 1.8$ & 121.4 & $144.6 \pm 11.3$ & $38.4 \pm 4.1$ & $95.2 \pm 7.8$ & $29.8 \pm 2.4$ & 77.0 & $* *$ \\
\hline K038 & $120.9 \pm 2.2$ & $85.4 \pm 1.2$ & $137.9 \pm 2.4$ & $100.5 \pm 1.8$ & 111.2 & $47.8 \pm 9.4$ & $47.4 \pm 4.1$ & $37.2 \pm 7.8$ & $19.4 \pm 2.4$ & 37.9 & $* *$ \\
\hline Mean & 121.6 & 91.6 & 139.4 & 104.7 & 114.3 & 91.5 & 80.6 & 75.4 & 46.8 & 73.6 & \\
\hline Minimum & 114.9 & 85.0 & 134.4 & 93.8 & 108.7 & 47.8 & 38.4 & 35.9 & 19.3 & 37.9 & \\
\hline Maximum & 127.5 & 101.6 & 149.2 & 119.0 & 123.4 & 208.1 & 152.5 & 181.6 & 89.6 & 139.1 & \\
\hline
\end{tabular}

Note. ${ }^{* *}$ - significant at $1 \%, *$ - significant at $5 \%$ and NS - non-significant at $5 \%$ level of significant, ${ }^{\circ}$ - Genotypes were analysed using missing block and hence they have the same values for all traits.

\subsubsection{Seed Yield (SY)}

The genotypes and genotype by environment interactions indicated highly significant variation $(\mathrm{p}<0.004)$ for seed yield $\mathrm{ha}^{-1}$ (Table 4). The evaluated genotypes recorded higher seed yield $\mathrm{ha}^{-1}$ in the long rain seasons compared to the short rain seasons at both sites, except ICCV 05312, ICCV 05315, ICCV 07306, ICCV 07308, ICCV 07313, ICCV 08302, ICCV 08303, ICCV 08307, ICCV 92318, K017, K038, K022, and K025 which recorded higher values in the short rain season at Kabete. During the long rain season, studied genotypes recorded higher seed yield ha $^{-1}$ at Juja compared to Kabete, except ICC 15802, ICCV 00402, IG 71055 and ICCV 92311. In the short rain season, higher seed yield ha ${ }^{-1}$ was recorded at Kabete except for ICC 13283, ICC 13461, ICCV 03404, ICCV 92311, ICCV 95311 and IG 71055 which recorded higher values at Juja. The ranking of genotypes for seed yield ha ${ }^{-1}$ varied across environments. Seven and thirty seven genotypes surpassed the check varieties ICCV 00305 and ICCV 92318 for seed yield ha ${ }^{-1}$ respectively which indicated considerable variation among the evaluated kabuli germplasm.

Based on overall means, the results indicated a wide range of variation among genotypes for seed yield ha ${ }^{-1}$. Eight genotypes were classified as low $(<0.8$ ton), 21 genotypes as moderate $(0.8-1.0$ ton $)$ and 10 genotypes were high ( $>1.0$ ton) yielder. Genotype ICCV 05315 gave the highest seed yield ha ${ }^{-1}(1.3$ ton) followed by ICC 13461 (1.3 ton), ICCV 07313 (1.2 ton), ICC 13764 (1.1 ton) and ICCV 00302 (1.1 ton). Genotypes K038 (0.4 ton), ICC 13283 ( 0.5 ton), ICCV 92318 (0.6 ton), ICCV 08307 ( 0.6 ton) and K025 (0.6 ton) recorded the lowest seed yield ha ${ }^{-1}$ (Table 4). The check varieties ICCV 00305 (1.0 ton) and ICCV 92318 (0.6 ton) were classified as moderate and low respectively for seed yield ha ${ }^{-1}$. Based on environment wise means, the highest seed yield ha ${ }^{-1}$ was recorded during the long rain season Juja at (1.4 ton) followed by long rain season at Kabete ( 0.9 ton), short rain season at Kabete (0.8) and short rain season at Juja (0.5) with a grand mean of 0.9 ton. 


\subsubsection{Hundred Seed Weight (100 seeds/g)}

There were highly significant $(\mathrm{p}<0.003)$ differences among genotypes and genotype by environment interactions for hundred seed weight (Table 4). Many genotypes, except ICC 13283, ICC 15802, ICCV 05312, ICCV 03404, ICCV 07306, ICCV 07313, ICCV 92311, ICCV 00402, ICCV 00305, ICCV 92318, IG 72109, K017, K022 and K038 recorded higher hundred seed weight in the short rain season compared to the long rain season at Juja. While at Kabete, most genotypes recorded higher hundred seed weight during the long rain season, except ICCV 05312, ICCV 07308, ICCV 07313, ICCV 08303, ICCV 08307, K022 and K038 compared to the short rain season. The studied genotypes recorded higher hundred seed weight at Kabete compared to Juja in both seasons. Thirty two and thirteen genotypes exceeded the check varieties ICCV 00305 and ICCV 92318 for hundred seed weight respectively.

On the basis of an overall means, the studied genotypes showed wide variability for hundred seed weight. Five genotypes were classified as low $(<25 \mathrm{~g}), 21$ genotypes as moderate $(25.0-35.0 \mathrm{~g})$ and 13 genotypes had high (> 35 g) 100 seed weight. Genotypes K025 (48.4 g), ICCV 08302 (45.3 g), ICCV 08303 (44.6 g), K041 (44.3 g) and ICCV 07308 (43.7 g) gave the highest 100 seed weight. Genotype ICC 13764 (15.7 g) followed by ICC $13461(17.4 \mathrm{~g})$, IG $71055(21.6 \mathrm{~g})$ and ICC $2482(22.2 \mathrm{~g})$ recorded the lowest 100 seed weight. The check varieties ICCV 00305 (26.5 g) and ICCV 92318 (34.1 g) were classified as moderate for 100 seed weight. With respect to environment wise means, the highest 100 seed weight was recorded in the long rain season at Kabete $(37.0 \mathrm{~g})$ followed by short rain season at Kabete $(35.7 \mathrm{~g})$, short rain season at Juja $(30.1 \mathrm{~g})$ and long rain season at Juja $(29.8 \mathrm{~g})$ with a grand mean of $33.1 \mathrm{~g}$.

Table 4. Performance Kabuli genotypes for seed yield ha ${ }^{-1}$ and hundred seed weight at Juja and Kabete in 2013

\begin{tabular}{|c|c|c|c|c|c|c|c|c|c|c|c|}
\hline \multirow{4}{*}{ Genotypes } & \multicolumn{4}{|c|}{ Seed Yield (SY) in tons $\mathrm{ha}^{-1}$} & \multirow{4}{*}{$\begin{array}{l}\text { Overall } \\
\text { Mean }\end{array}$} & \multicolumn{4}{|c|}{ Hundred Seed Weight (HSW) in gram } & \multirow{4}{*}{$\begin{array}{l}\text { Overall } \\
\text { Mean }\end{array}$} & \multirow{4}{*}{$\begin{array}{l}\mathrm{P} . \\
\text { value }\end{array}$} \\
\hline & Long Rain & Short & Long Rain & Short Rain & & Long Rain & Short & Long Rain & Short Rain & & \\
\hline & Juja & Rain Juja & Kabete & Kabete & & Juja & Rain Juja & Kabete & Kabete & & \\
\hline & Mean \pm S.E & Mean \pm S.E & Mean \pm S.E & Mean \pm S.E & & Mean \pm S.E & Mean \pm S.E & Mean \pm S.E & Mean \pm S.E & & \\
\hline ICCV 05315 & $2.3 \pm 0.0$ & $0.4 \pm 0.0$ & $1.1 \pm 0.0$ & $1.5 \pm 0.0$ & 1.3 & $37.4 \pm 1.4$ & $37.6 \pm 1.0$ & $44.5 \pm 1.2$ & $44.4 \pm 1.2$ & 41.0 & $* *$ \\
\hline ICC 13461 & $2.3 \pm 0.0$ & $0.6 \pm 0.0$ & $2.0 \pm 0.0$ & $0.4 \pm 0.0$ & 1.3 & $13.9 \pm 1.4$ & $15.0 \pm 1.0$ & $22.0 \pm 1.2$ & $18.5 \pm 1.2$ & 17.4 & $* *$ \\
\hline ICCV 07313 & $2.2 \pm 0.0$ & $0.3 \pm 0.0$ & $0.8 \pm 0.0$ & $1.5 \pm 0.0$ & 1.2 & $38.7 \pm 1.4$ & $37.8 \pm 1.0$ & $44.9 \pm 1.2$ & $47.7 \pm 1.2$ & 42.3 & $* *$ \\
\hline ICC 13764 & $1.8 \pm 0.0$ & $0.5 \pm 0.0$ & $1.6 \pm 0.0$ & $0.7 \pm 0.0$ & 1.1 & $12.4 \pm 1.4$ & $13.6 \pm 1.0$ & $19.5 \pm 1.2$ & $17.1 \pm 1.2$ & 15.7 & $* *$ \\
\hline ICCV 00302 & $1.7 \pm 0.0$ & $0.7 \pm 0.0$ & $1.4 \pm 0.0$ & $0.7 \pm 0.0$ & 1.1 & $24.9 \pm 1.4$ & $25.4 \pm 1.0$ & $31.1 \pm 1.2$ & $30.1 \pm 1.2$ & 27.9 & $* *$ \\
\hline ICC 2482 & $2.1 \pm 0.0$ & $0.4 \pm 0.0$ & $1.2 \pm 0.0$ & $0.6 \pm 0.0$ & 1.1 & $16.3 \pm 1.6$ & $19.8 \pm 1.0$ & $25.3 \pm 1.5$ & $27.3 \pm 1.2$ & 22.2 & $* *$ \\
\hline ICCV 03404 & $1.2 \pm 0.0$ & $1.3 \pm 0.0$ & $0.9 \pm 0.0$ & $0.7 \pm 0.0$ & 1.1 & $35.1 \pm 1.4$ & $34.6 \pm 1.0$ & $42.2 \pm 1.2$ & $36.1 \pm 1.2$ & 37.0 & $* *$ \\
\hline ICCV 00402 & $0.6 \pm 0.0$ & $0.5 \pm 0.0$ & $1.3 \pm 0.0$ & $1.7 \pm 0.0$ & 1.0 & $22.2 \pm 1.4$ & $20.8 \pm 1.0$ & $33.6 \pm 1.2$ & $32.9 \pm 1.2$ & 27.4 & $* *$ \\
\hline ICCV 07306 & $1.3 \pm 0.0$ & $0.5 \pm 0.0$ & $0.5 \pm 0.0$ & $1.8 \pm 0.0$ & 1.0 & $31.9 \pm 1.4$ & $31.7 \pm 1.0$ & $42.7 \pm 1.2$ & $38.7 \pm 1.2$ & 36.2 & $* *$ \\
\hline $\begin{array}{l}\text { ICCV } 00305 \\
\text { (Check) }\end{array}$ & $1.6 \pm 0.0$ & $0.4 \pm 0.0$ & $1.4 \pm 0.0$ & $0.7 \pm 0.0$ & 1.0 & $23.0 \pm 1.4$ & $20.8 \pm 1.0$ & $31.7 \pm 1.2$ & $30.2 \pm 1.2$ & 26.5 & $* *$ \\
\hline ICCV 05312 & $1.4 \pm 0.0$ & $0.7 \pm 0.0$ & $0.6 \pm 0.0$ & $1.2 \pm 0.0$ & 1.0 & $36.1 \pm 1.4$ & $35.3 \pm 1.0$ & $41.5 \pm 1.2$ & $42.3 \pm 1.2$ & 38.8 & $* *$ \\
\hline IG 71055 & $1.0 \pm 0.0$ & $0.7 \pm 0.0$ & $1.7 \pm 0.0$ & $0.4 \pm 0.0$ & 1.0 & $19.6 \pm 1.4$ & $20.1 \pm 1.0$ & $27.2 \pm 1.2$ & $19.5 \pm 1.2$ & 21.6 & $* *$ \\
\hline ICCV 08302 & $1.4 \pm 0.0$ & $0.4 \pm 0.0$ & $1.0 \pm 0.0$ & $1.1 \pm 0.0$ & 1.0 & $42.3 \pm 1.4$ & $44.4 \pm 1.0$ & $49.4 \pm 1.2$ & $45.1 \pm 1.2$ & 45.3 & $* *$ \\
\hline ICCV 92311 & $1.2 \pm 0.0$ & $0.5 \pm 0.0$ & $1.6 \pm 0.0$ & $0.4 \pm 0.0$ & 0.9 & $33.7 \pm 1.4$ & $33.5 \pm 1.0$ & $41.6 \pm 1.2$ & $34.4 \pm 1.2$ & 35.8 & $* *$ \\
\hline ICC 15802 & $1.2 \pm 0.0$ & $0.4 \pm 0.0$ & $1.7 \pm 0.0$ & $0.6 \pm 0.0$ & 0.9 & $20.6 \pm 1.4$ & $20.4 \pm 1.0$ & $29.4 \pm 1.2$ & $25.0 \pm 1.2$ & 23.8 & $* *$ \\
\hline ICCV 07308 & $1.8 \pm 0.0$ & $0.5 \pm 0.0$ & $0.5 \pm 0.0$ & $1.0 \pm 0.0$ & 0.9 & $41.1 \pm 1.4$ & $42.7 \pm 1.0$ & $45.0 \pm 1.5$ & $45.8 \pm 1.2$ & 43.6 & $* *$ \\
\hline ICC $10885^{\circ}$ & $1.4 \pm 0.0$ & $0.5 \pm 0.0$ & $0.9 \pm 0.0$ & $0.8 \pm 0.0$ & 0.9 & $29.8 \pm 9.4$ & $30.1 \pm 9.4$ & $37.0 \pm 9.4$ & $35.7 \pm 9.4$ & 33.1 & $* *$ \\
\hline ICC $13357^{\circ}$ & $1.4 \pm 0.0$ & $0.5 \pm 0.0$ & $0.9 \pm 0.0$ & $0.8 \pm 0.0$ & 0.9 & $29.8 \pm 9.4$ & $30.1 \pm 9.4$ & $37.0 \pm 9.4$ & $35.7 \pm 9.4$ & 33.1 & $* *$ \\
\hline ICC $16654^{\circ}$ & $1.4 \pm 0.0$ & $0.5 \pm 0.0$ & $0.9 \pm 0.0$ & $0.8 \pm 0.0$ & 0.9 & $29.8 \pm 9.4$ & $30.1 \pm 9.4$ & $37.0 \pm 9.4$ & $35.7 \pm 9.4$ & 33.1 & $* *$ \\
\hline $\operatorname{ICC} 7315^{\circ}$ & $1.4 \pm 0.0$ & $0.5 \pm 0.0$ & $0.9 \pm 0.0$ & $0.8 \pm 0.0$ & 0.9 & $29.8 \pm 9.4$ & $30.1 \pm 9.4$ & $37.0 \pm 9.4$ & $35.7 \pm 9.4$ & 33.1 & $* *$ \\
\hline
\end{tabular}




\begin{tabular}{|c|c|c|c|c|c|c|c|c|c|c|c|}
\hline ICCV $03309^{\circ}$ & $1.4 \pm 0.0$ & $0.5 \pm 0.0$ & $0.9 \pm 0.0$ & $0.8 \pm 0.0$ & 0.9 & $29.8 \pm 9.4$ & $30.1 \pm 9.4$ & $37.0 \pm 9.4$ & $35.7 \pm 9.4$ & 33.1 & $* *$ \\
\hline ICCV $06304^{\circ}$ & $1.4 \pm 0.0$ & $0.5 \pm 0.0$ & $0.9 \pm 0.0$ & $0.8 \pm 0.0$ & 0.9 & $29.8 \pm 9.4$ & $30.1 \pm 9.4$ & $37.0 \pm 9.4$ & $35.7 \pm 9.4$ & 33.1 & $* *$ \\
\hline $\operatorname{ICCV} 08310^{\circ}$ & $1.4 \pm 0.0$ & $0.5 \pm 0.0$ & $0.9 \pm 0.0$ & $0.8 \pm 0.0$ & 0.9 & $29.8 \pm 9.4$ & $30.1 \pm 9.4$ & $37.0 \pm 9.4$ & $35.7 \pm 9.4$ & 33.1 & $* *$ \\
\hline ICCV $07304^{\circ}$ & $1.4 \pm 0.0$ & $0.5 \pm 0.0$ & $0.9 \pm 0.0$ & $0.8 \pm 0.0$ & 0.9 & $29.8 \pm 9.4$ & $30.1 \pm 9.4$ & $37.0 \pm 9.4$ & $35.7 \pm 9.4$ & 33.1 & $* *$ \\
\hline $\mathrm{K} 004^{\circ}$ & $1.4 \pm 0.0$ & $0.5 \pm 0.0$ & $0.9 \pm 0.0$ & $0.8 \pm 0.0$ & 0.9 & $29.8 \pm 9.4$ & $30.1 \pm 9.4$ & $37 \pm 9.4$ & $35.7 \pm 9.4$ & 33.1 & $* *$ \\
\hline $\mathrm{K} 012^{\circ}$ & $1.4 \pm 0.0$ & $0.5 \pm 0.0$ & $0.9 \pm 0.0$ & $0.8 \pm 0.0$ & 0.9 & $29.8 \pm 9.4$ & $30.1 \pm 9.4$ & $37.0 \pm 9.4$ & $35.7 \pm 9.4$ & 33.1 & $* *$ \\
\hline ICCV $08313^{\circ}$ & $1.4 \pm 0.0$ & $0.5 \pm 0.0$ & $0.9 \pm 0.0$ & $0.8 \pm 0.0$ & 0.9 & $29.8 \pm 9.4$ & $30.1 \pm 9.4$ & $37.0 \pm 9.4$ & $35.7 \pm 9.4$ & 33.1 & $* *$ \\
\hline $\mathrm{K} 034^{\circ}$ & $1.4 \pm 0.0$ & $0.5 \pm 0.0$ & $0.9 \pm 0.0$ & $0.8 \pm 0.0$ & 0.9 & $29.8 \pm 9.4$ & $30.1 \pm 9.4$ & $37.0 \pm 9.4$ & $35.7 \pm 9.4$ & 33.1 & $* *$ \\
\hline IG 72109 & $2.1 \pm 0.0$ & $0.5 \pm 0.0$ & $0.6 \pm 0.0$ & $0.5 \pm 0.0$ & 0.9 & $23.6 \pm 1.4$ & $22.0 \pm 1.0$ & $31.7 \pm 1.2$ & $30.3 \pm 1.2$ & 26.9 & $* *$ \\
\hline ICCV 08303 & $1.2 \pm 0.0$ & $0.4 \pm 0.0$ & $0.5 \pm 0.0$ & $1.4 \pm 0.0$ & 0.9 & $40.9 \pm 1.4$ & $41.5 \pm 1.0$ & $47.4 \pm 1.2$ & $48.8 \pm 1.2$ & 44.6 & $* *$ \\
\hline ICCV 95311 & $1.5 \pm 0.0$ & $0.6 \pm 0.0$ & $0.8 \pm 0.0$ & $0.4 \pm 0.0$ & 0.8 & $26.3 \pm 1.4$ & $27.0 \pm 1.0$ & $37.9 \pm 1.2$ & $29.2 \pm 1.2$ & 30.1 & $* *$ \\
\hline K041 & $1.0 \pm 0.0$ & $0.4 \pm 0.0$ & $0.9 \pm 0.0$ & $0.7 \pm 0.0$ & 0.7 & $39.9 \pm 1.4$ & $43.4 \pm 1.0$ & $47.0 \pm 1.2$ & $46.8 \pm 1.2$ & 44.3 & $* *$ \\
\hline K022 & $1.3 \pm 0.0$ & $0.4 \pm 0.0$ & $0.4 \pm 0.0$ & $0.7 \pm 0.0$ & 0.7 & $33.4 \pm 1.6$ & $33.3 \pm 1.0$ & $37.1 \pm 1.2$ & $43.8 \pm 1.2$ & 36.9 & $* *$ \\
\hline K017 & $1.0 \pm 0.0$ & $0.4 \pm 0.0$ & $0.2 \pm 0.0$ & $1.2 \pm 0.0$ & 0.7 & $29.9 \pm 1.4$ & $28.4 \pm 1.0$ & $40.1 \pm 1.5$ & $37.2 \pm 1.2$ & 33.9 & $* *$ \\
\hline K025 & $1.0 \pm 0.0$ & $0.4 \pm 0.0$ & $0.4 \pm 0.0$ & $0.6 \pm 0.0$ & 0.6 & $44.3 \pm 1.6$ & $45.8 \pm 1.0$ & $51.4 \pm 1.2$ & $50.9 \pm 1.2$ & 48.1 & $* *$ \\
\hline ICCV 08307 & $0.7 \pm 0.0$ & $0.4 \pm 0.0$ & $0.4 \pm 0.0$ & $0.7 \pm 0.0$ & 0.6 & $33.7 \pm 1.4$ & $34.6 \pm 1.0$ & $38.3 \pm 1.2$ & $40.3 \pm 1.2$ & 36.7 & $* *$ \\
\hline $\begin{array}{l}\text { ICCV } 92318 \\
\text { (Check) }\end{array}$ & $1.0 \pm 0.0$ & $0.3 \pm 0.0$ & $0.4 \pm 0.0$ & $0.7 \pm 0.0$ & 0.6 & $31.4 \pm 1.4$ & $31.0 \pm 1.0$ & $37.1 \pm 1.2$ & $37.0 \pm 1.2$ & 34.1 & $* *$ \\
\hline ICC 13283 & $0.9 \pm 0.0$ & $0.3 \pm 0.0$ & $0.5 \pm 0.0$ & $0.3 \pm 0.0$ & 0.5 & $23.3 \pm 1.6$ & $23.0 \pm 1.0$ & $32.0 \pm 1.2$ & $25.9 \pm 1.2$ & 26.0 & $* *$ \\
\hline K038 & $0.6 \pm 0.0$ & $0.2 \pm 0.0$ & $0.3 \pm 0.0$ & $0.5 \pm 0.0$ & 0.4 & $29.5 \pm 1.4$ & $28.4 \pm 1.0$ & $25.9 \pm 1.2$ & $37.1 \pm 1.2$ & 30.2 & $* *$ \\
\hline Means & 1.4 & 0.5 & 0.9 & 0.8 & 0.9 & 29.8 & 30.1 & 37.0 & 35.7 & 33.1 & \\
\hline Minimum & 0.6 & 0.2 & 0.2 & 0.3 & 0.4 & 12.4 & 13.6 & 19.5 & 17.1 & 15.7 & \\
\hline Maximum & 2.3 & 1.3 & 2.0 & 1.8 & 1.3 & 44.3 & 45.8 & 51.4 & 50.9 & 48.1 & \\
\hline
\end{tabular}

Note. ${ }^{* *}$ - significant at $1 \%,{ }^{*}$ - significant at $5 \%$ and NS - non-significant at $5 \%$ level of significant, ${ }^{\circ}$ - Genotypes were analysed using missing block and hence they have the same values for all traits.

\subsection{Qualitative Characters of Kabuli Chickpea Genotypes}

Eight qualitative traits showed a wide range of variation among studied Kabuli chickpea genotypes (Figure 1) for all characters, except for leaf type (normal) and pod dehiscence (non-dehiscence) for which the genotypes were monomorphic. Genotypes were polymorphic with respect to stem pigmentation and were classified as dark green (59\%), light green (38.0\%) and partly purple (3.0\%) (Figure 1A). The results indicated four types of growth habit namely semi erect, semi- spreading, spreading and prostrate. Semi spreading $(46.0 \%)$ growth habit recorded the highest frequency followed by spreading $(28.0 \%)$ and semi- erect $(21.0 \%)$ while prostrate $(5.0 \%)$ growth habit recorded the lowest frequency (Figure 1B). Evaluated genotypes also showed variations for seed testa texture such as smooth $(79.0 \%)$, tuberculated $(18.0 \%)$ and rough $(3.0 \%)$ (Figure 1C). There were considerable differences among genotypes for flower colour such as white (97.0\%) and light pink (3.0\%) (Figure 1D). The evaluated genotypes also showed wide variation with respect to seed shape such as owl's head $(92.0 \%)$, pea shaped $(5.0 \%)$ and angular (3.0\%) (Figure 1E). The studied genotypes further showed variability for seed coat colour like creamy white (97\%) and brown (3\%) (Figure 1F). 
ఐ Light Green $\quad$ Dark Green $\quad$ Partly Purple

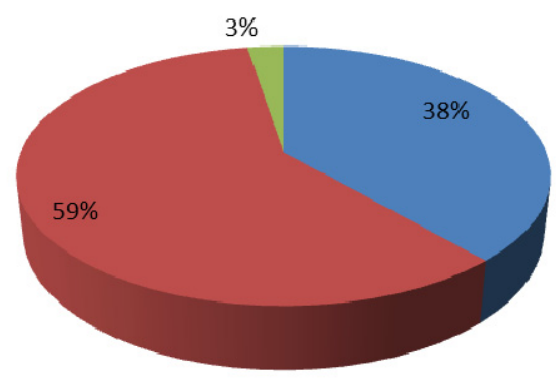

A. Stem Pigmentation

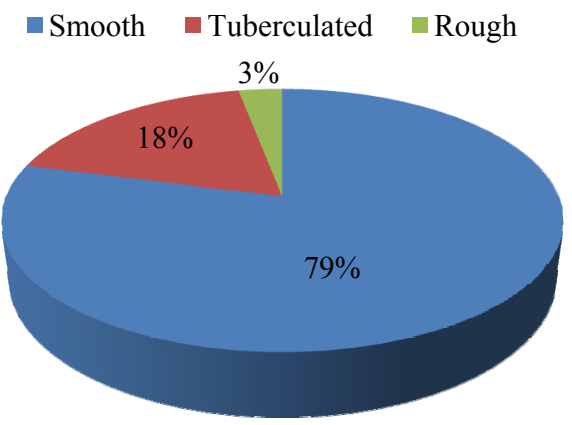

C. Seed Testa Texture

$\square$ Owl's head $\square$ Pea-shaped $\quad$ Angular

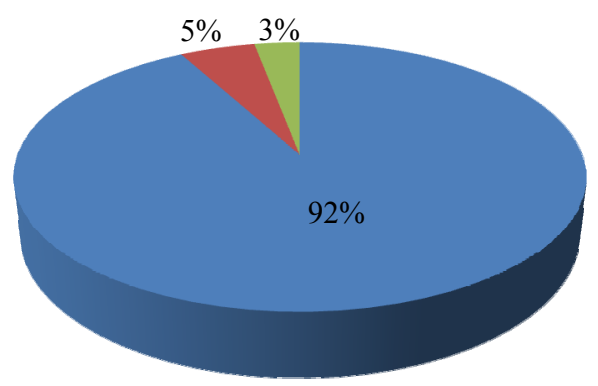

E. Seed Shape

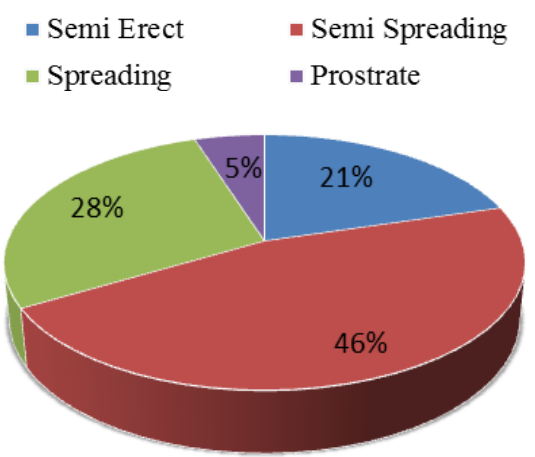

B. Growth Habit

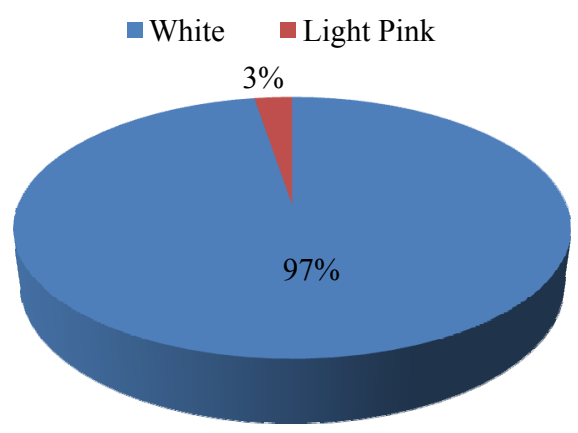

D. Flower Colour

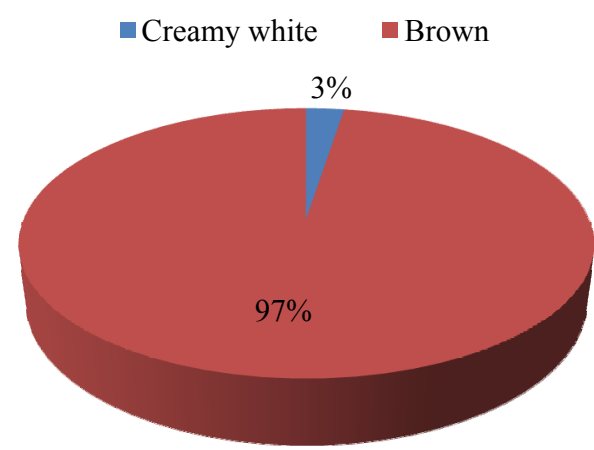

F. Seed Coat Colour

Figure 1. Distribution of qualitative characters of Kabuli chickpea genotypes at Juja and Kabete in 2013

\subsection{Association among Quantitative Traits of Kabuli Chickpea Genotypes}

The correlation coefficients among quantitative traits are presented in Table 5. There was positive and significant $(\mathrm{p}<0.05)$ correlation of seed yield $\mathrm{ha}^{-1}$ with biomass yield ha ${ }^{-1}\left(\mathrm{r}=0.85^{* *}\right)$, number of pods $\operatorname{plant}^{-1}\left(\mathrm{r}=0.57^{* *}\right)$, plant canopy width $\left(\mathrm{r}=0.39^{* *}\right)$ and number of secondary branches plant ${ }^{-1}\left(\mathrm{r}=0.34^{*}\right)$ but non-significantly correlated with the other characters.

Biomass yield $\mathrm{ha}^{-1}$ indicated positive and significant $(\mathrm{p}<0.05)$ correlation with days to $50 \%$ flowering $(\mathrm{r}=$ $\left.0.39^{* *}\right)$, days to $50 \%$ podding $\left(\mathrm{r}=0.38^{*}\right)$, plant canopy width $\left(\mathrm{r}=0.60^{* *}\right)$, plant height $\left(\mathrm{r}=0.38^{*}\right)$, number of 
primary branches plant ${ }^{-1}\left(\mathrm{r}=0.55^{* *}\right)$, number of secondary branches plant ${ }^{-1}\left(\mathrm{r}=0.56^{* *}\right)$, number of pods plant ${ }^{-1}$ $\left(\mathrm{r}=0.75^{* *}\right)$ and days to $75 \%$ maturity $\left(\mathrm{r}=0.53^{* *}\right)$. It was negatively and highly significantly $(\mathrm{p}<0.01)$ correlated with 100 seed weight $\left(\mathrm{r}=-0.53^{* *}\right)$ but non-significantly correlated with the other traits.

Number of pods plant ${ }^{-1}$ showed positive and highly significant $(\mathrm{p}<0.01)$ correlation with days to $50 \%$ flowering $\left(\mathrm{r}=0.65^{* *}\right)$, days to $50 \%$ podding $\left(\mathrm{r}=0.71^{* *}\right)$, plant canopy width $\left(\mathrm{r}=0.68^{* *}\right)$, plant height $\left(\mathrm{r}=0.56^{* *}\right)$, number of primary branches $\operatorname{plant}^{-1}\left(\mathrm{r}=0.74^{* *}\right)$, number of secondary branches plant ${ }^{-1}\left(\mathrm{r}=0.78^{* *}\right)$, days to $75 \%$ maturity $\left(r=0.79^{* *}\right)$ and negatively significant correlation with pod filling period $\left(r=-0.50^{* *}\right)$ and 100 seed weight $\left(\mathrm{r}=-0.70^{* *}\right)$.

There was positive and highly significant $(\mathrm{p}<0.01)$ correlation of days to $75 \%$ maturity with days to $50 \%$ flowering $\left(\mathrm{r}=0.83^{* *}\right)$, days to $50 \%$ podding $\left(\mathrm{r}=0.89^{* *}\right)$, plant canopy width $\left(\mathrm{r}=0.63^{* *}\right)$, plant height $(\mathrm{r}=$ $0.62 * *)$, number of primary branches $\operatorname{plant}^{-1}\left(\mathrm{r}=0.81^{* *}\right)$ and number of secondary branches plant ${ }^{-1}\left(\mathrm{r}=0.78^{* *}\right)$. Day to $75 \%$ maturity was negatively significant correlated with pod filling period $(r=-0.63 * *)$ and 100 seed weight $\left(r=-0.78^{* *}\right)$ but non-significant correlation with other traits.

Days to $50 \%$ podding was positively and highly significantly $(\mathrm{p}<0.01)$ correlated with days to $50 \%$ flowering $(\mathrm{r}$ $\left.=0.96^{* *}\right)$. Plant height also showed positive and highly significant $(\mathrm{p}<0.01)$ correlation with days to $50 \%$ flowering $\left(\mathrm{r}=0.45^{* *}\right)$, days to $50 \%$ podding $\left(\mathrm{r}=0.50^{* *}\right)$ and plant canopy width $\left(\mathrm{r}=0.77^{* *}\right)$.

Table 5. Correlation coefficient among quantitative characters of Kabuli chickpea genotypes at Juja and Kabete in 2013

\begin{tabular}{|c|c|c|c|c|c|c|c|c|c|c|c|c|c|}
\hline & DF & DP & PCW & $\mathrm{PH}$ & NPB & NSB & DM & PFP & $\mathrm{NPP}^{-1}$ & $\mathrm{NSP}^{-1}$ & $\mathrm{BM}$ & SY & HSW \\
\hline $\mathrm{DF}$ & 1 & & & & & & & & & & & & \\
\hline DP & $0.96 * *$ & 1 & & & & & & & & & & & \\
\hline $\mathrm{PCW}$ & $0.62 * *$ & $0.61 * *$ & 1 & & & & & & & & & & \\
\hline $\mathrm{PH}$ & $0.45^{* *}$ & $0.50 * *$ & $0.77 * *$ & 1 & & & & & & & & & \\
\hline NPB & $0.77 * *$ & $0.78 * *$ & $0.78^{* *}$ & $0.72 * *$ & 1 & & & & & & & & \\
\hline NSB & $0.72 * *$ & $0.75^{* *}$ & $0.72 * *$ & $0.69^{* *}$ & $0.88^{* *}$ & 1 & & & & & & & \\
\hline DM & $0.83 * *$ & $0.89 * *$ & $0.63 * *$ & $0.62 * *$ & $0.81^{* *}$ & $0.78^{* *}$ & 1 & & & & & & \\
\hline PFP & $-0.95 * *$ & $-0.88 * *$ & $-0.54 * *$ & $-0.30 *$ & $-0.66 * *$ & $-0.61 * *$ & $-0.63 * *$ & 1 & & & & & \\
\hline $\mathrm{NPP}^{-1}$ & $0.65^{* *}$ & $0.71 * *$ & $0.68^{* *}$ & $0.56^{* *}$ & $0.74 * *$ & $0.78 * *$ & $0.79 * *$ & $-0.50^{* *}$ & 1 & & & & \\
\hline $\mathrm{NSP}^{-1}$ & $0.19 \mathrm{NS}$ & $0.17 \mathrm{NS}$ & $0.39 * *$ & $0.31^{*}$ & $0.25 \mathrm{NS}$ & $0.23 \mathrm{NS}$ & $0.18 \mathrm{NS}$ & $-0.20 \mathrm{NS}$ & $0.11 \mathrm{NS}$ & 1 & & & \\
\hline $\mathrm{BM}$ & $0.39 * *$ & $0.38^{*}$ & $0.60^{* *}$ & $0.38^{*}$ & $0.55^{* *}$ & $0.56^{* *}$ & $0.53 * *$ & $-0.28 \mathrm{NS}$ & $0.75 * *$ & $0.09 \mathrm{NS}$ & 1 & & \\
\hline SY & $0.08 \mathrm{NS}$ & $0.06 \mathrm{NS}$ & $0.39 * *$ & $0.12 \mathrm{NS}$ & $0.29 \mathrm{NS}$ & $0.34 *$ & $0.22 \mathrm{NS}$ & $0.00 \mathrm{NS}$ & $0.57 * *$ & $-0.03 \mathrm{NS}$ & $0.85^{* *}$ & 1 & \\
\hline HSW & $-0.81 * *$ & -0.82 & $-0.60^{* *}$ & $-0.41 * *$ & $-0.64 * *$ & $-0.62 * *$ & $-0.78 * *$ & $0.71 * *$ & $-0.70^{* *}$ & $-0.22 \mathrm{NS}$ & $-0.53 * *$ & $-0.22 \mathrm{NS}$ & 1 \\
\hline
\end{tabular}

Note. **- significant at $1 \%$ level of significant (1. o. s) $(\operatorname{Pr}<0.01) ; *$ - significant at $5 \%$ level of significant $(\operatorname{Pr}<$ $0.05)$ and NS - non-significant at 5\% ( $\mathrm{Pr}>0.05)$; DF - days to 50\% flowering; DP - days to 50\% podding; PCW plant canopy width; PH - plant height; NPB - number of primary branches plant ${ }^{-1}$; NSB - number of secondary branches plant ${ }^{-1}$; DM - days to $75 \%$ maturity; PFP - pod filling period; NPP ${ }^{-1}$ number of pods plant ${ }^{-1}$; $\mathrm{NSP}^{-1}$ Number of seeds pod ${ }^{-1}, \mathrm{BM}$ - biomass yield ha ${ }^{-1}$, SY - seed yield ha ${ }^{-1}$ and HSW - hundred seed weight.

\subsection{Principal Component Analysis}

The results on principal component analysis showed the first three components (PCs) with eigenvalues greater than one explained significant proportion of the total variations. The first three principal components accounted for $80.14 \%$ total variance in the studied characters. The other principal components with less than one eigenvalues have not been included and explained in this paper. The proportions of cumulative variance explained by each of the first three principal components were $56.96 \%, 69.57 \%$ and 80.14 respectively. The characters associated with the first three principal components, their eigenvalues, variances percentage, cumulative proportion variances are given in Table 6. Besides, eigenvalues of the seven principal components are provided as scree plot in Figure 2. The first principal component explained 56.96\% of the total variations and 
was positively associated with the days to $50 \%$ flowering, days to $50 \%$ podding, plant canopy width, plant height, number of primary and secondary branches plant ${ }^{-1}$, days to $75 \%$ maturity, number of pods plant ${ }^{-1}$ and biomass yield $\mathrm{ha}^{-1}$. While pod length and pod filling period contributed in negative direction. The second principal component, which contributed to the total variations of $12.61 \%$ and was positively contributed through the number of leaflet leaf ${ }^{1}$ at podding, plant canopy width, plant height, pod filling period, number of pods plant $^{-1}$, biomass yield $\mathrm{ha}^{-1}$ and seed yield $\mathrm{ha}^{-1}$. In negative direction, it was related with days to $50 \%$ flowering and days to $50 \%$ podding. The third principal component explained $10.57 \%$ of the total variations. The third component was predominantly and positively associated with differences in number of pods plant ${ }^{-1}$, biomass yield ha ${ }^{-1}$ and seed yield ha ${ }^{-1}$. While in negative direction, it was mainly associated with number of leaflet leaf ${ }^{1}$ at podding, plant canopy width, pod length, plant height and number of seeds $\operatorname{pod}^{-1}$.

Table 6. Loading vectors for the first three principal components of characters associated with agronomic and yield performances

\begin{tabular}{|c|c|c|c|}
\hline Characters & Principal Component 1 & Principal Component 2 & Principal Component 3 \\
\hline Days to $50 \%$ flowering & 0.31 & -0.27 & 0.02 \\
\hline Days to $50 \%$ Podding & 0.31 & -0.28 & -0.06 \\
\hline Number of Leaflet Leaf ${ }^{1}$ at Podding & 0.13 & 0.23 & -0.55 \\
\hline Plant Canopy Width & 0.28 & 0.19 & -0.20 \\
\hline Pod Length & -0.25 & 0.13 & -0.20 \\
\hline Plant Height & 0.23 & 0.15 & -0.31 \\
\hline Number of Primary Branches Plant ${ }^{-1}$ & 0.30 & -0.04 & -0.07 \\
\hline Number of Secondary Branches Plant ${ }^{-1}$ & 0.31 & 0.04 & 0.05 \\
\hline Days to $75 \%$ Maturity & 0.31 & -0.08 & 0.07 \\
\hline Pod Filling Period & -0.27 & 0.33 & 0.03 \\
\hline Number of Pods Plant ${ }^{-1}$ & 0.29 & 0.17 & 0.24 \\
\hline Number of Seeds Pod ${ }^{-1}$ & 0.11 & 0.06 & -0.56 \\
\hline Biomass Yield ha ${ }^{-1}$ & 0.22 & 0.46 & 0.20 \\
\hline Seed Yield ha ${ }^{-1}$ & 0.12 & 0.59 & 0.28 \\
\hline Hundred Seed Weight & -0.29 & 0.12 & -0.09 \\
\hline Eigenvalues & 8.54 & 1.89 & 1.59 \\
\hline Proportion of Variance (\%) & 56.96 & 12.61 & 10.57 \\
\hline Cumulative Variance (\%) & 56.96 & 69.57 & 80.14 \\
\hline
\end{tabular}

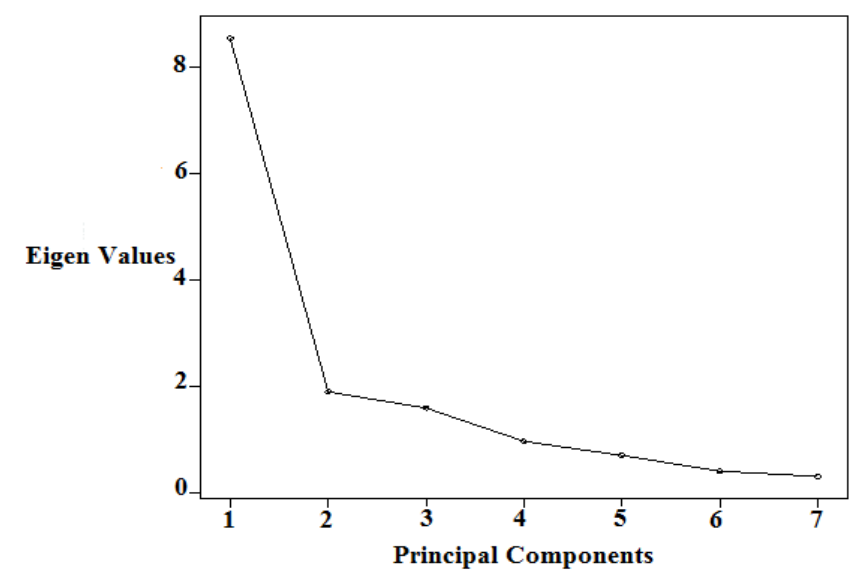

Figure 2. Scree plot constructed based on seven principal components and their eigen values 


\section{Discussion}

Crop genetic resources including chickpea germplasm represents an invaluable source of genetic diversity that is expected to be highly useful for current and future breeding programmes. A success in genetic improvement of chickpea greatly depends on the availability of genetic resources and the extent of genetic variations and interrelationship among important traits. Knowledge of genetic variations among genotypes and relationships of economic characters would assist chickpea breeders to devise suitable breeding strategies and to develop the most adaptive and productive varieties. In addition, analysis of agronomic and yield related traits and their contribution towards seed yield could provide a good opportunity for indirect selection of high yielding genotypes. The results from this study showed wide variations for all studied characters, which indicated the existence of considerable diversity. The diversity and interrelationship for the studied traits is described below.

Early flowering trait is an essential in a chickpea crop improvement. Flowering stage is highly sensitive and may be influenced by extreme temperatures due to adverse effect on viability of pollen and pollination which could result in poor fertilization and low seed set. The results from this study showed substantial differences among chickpea genotypes for days to 50\% flowering (Table 2). Likewise, Atta et al. (2008), Khan et al. (2011) and Tesfamichael et al. (2014) reported significant and wide variation for days to flowering in chickpea germplasm. The current results were not in agreement with those of Zelalem (2014) who reported non-significant difference for days to flowering in haricot bean. The documented information and identified early flowering genotypes from this study could be utilized by breeders as parent materials for hybridization in chickpea and develop early flowering varieties for the region.

Plant height is one of the desirable characters in a chickpea variety development. The evaluated genotypes showed wide variability with respect to plant height (Table 2). Similar results were reported by Parameshwarappa et al. (2012), Tesfamichael et al. (2014) in chickpea germplasm and Yimram et al. (2009) in mung bean germplasm. However, in contrary to these results Roy et al. (2013) reported non-significant difference for plant height in lentil. The available variations for plant height from the studied germplasm can be exploited in chickpea breeding programmes.

Earliness trait is one of the prime breeding goals of chickpea as most farmers generally seek for early maturing varieties in order to enable the crop to mature within the growing season and give reasonable yield. Upadhyaya et al. (2007) reported that early-maturity helps chickpea crop to escape terminal heat and drought and increases its adaptation especially in the sub-tropics. The current results showed significant difference for days to $75 \%$ maturity (Table 3). Similar findings were reported by Atta et al. (2008), Khan et al. (2011), Tesfamichael et al. (2014) in chickpea genotypes and Oladejo et al. (2011) in cowpea. The identified early maturing genotypes with reasonable yield traits could be utilized for development of early maturing kabuli varieties and hence contribute to the promotion of chickpea in the region particularly during the short rain seasons.

Plant growth behavior of legume crops including chickpea can be determined by number of filled pods plant ${ }^{-1}$. The studied genotypes showed existence of wide variation for number of pods plant ${ }^{-1}$ (Table 3 ). Similar results were reported by many researchers (Qureshi et al., 2004; Malik et al., 2010; Gul et al., 2013; Tesfamichael et al., 2014) in chickpea. The current study indicated that genotypes with higher number of pods plant ${ }^{-1}$ were well adapted to the climatic condition and gave more pods and these could be contributed to high ultimate yield ha ${ }^{-1}$. Therefore this study indicated there is a potential of obtaining high pod yielding genotypes from the evaluated kabuli germplasm. The obtained information and identified high pod yielding and promising genotypes could be exploited by chickpea breeders in the region to develop high yielding varieties suitable for semi-arid areas.

Yield is a quantitative variable trait and the result of several physiological and biochemical processes in the crop growth and development stages. The results from this investigation exhibited substantial differences among genotypes for seed yield ha- ${ }^{-1}$ (Table 4). The presence of wide genetic variations for seed yield ha ${ }^{-1}$ showed the potential of the evaluated kabuli germplasm to develop high yielding genotypes for specific and broad adaptation. Similarly in chickpea, previous studies have reported significant genetic variation for seed yield (Malik et al., 2009; Padmavathi et al., 2013; Tesfamichael et al., 2014).

High seed yield ha ${ }^{-1}$ is one of the main criteria for identifying and selecting superior varieties for most farmers. Thus, promising and high yielding genotypes from this germplasm could be used directly by the farmers or exploited for future hybridization of chickpea in the region.

There were highly significant differences among the evaluated genotypes for 100 seed weight (Table 4). These results were in agreement with those of Qureshi et al. (2004); Khan et al. (2011) and Malik et al. (2009) who reported significant difference for 100 seed weight in chickpea germplasm. 
Seed size is one of the most important yield related traits in chickpea crop and determines the final seed weight. The recorded variations for 100 seed weight could be attributed to small seed size and undeveloped seeds.

Overall, the results from this study showed significant and wide genetic variation among the evaluated genotypes with respect to all studied characters. This indicated that appreciable diversity existed within the kabuli chickpea germplasm and could be utilized by chickpea breeders in the region. The wide diversity could be attributed to genotype differences, environment and genotype by environment interactions. Genotypes ranking for most studied traits varied across environments. This indicated the presence of a crossover type of genotype by environment interactions. High yielding genotypes across environments identified in this study include ICCV 05315 (1.3 ton), ICC 13461 (1.3 ton), ICCV 07313 (1.3 ton), ICC 13764 (1.1 ton) and ICCV 00302 (1.1 ton). These promising varieties could be recommended for release to the farmers in the region especially in the semi-arid areas.

The studied qualitative characters showed wide variation among evaluated genotypes (Figure 1). Majority of the evaluated genotypes were semi-spreading and had creamy white seed coat colour, white flower colour, dark green pigmented stem, owl' head seed shape and smooth seed testa texture. The current results were in line with those of Ramanappa et al. (2013), Upadhyaya et al. (2002), (Qureshi et al., 2004) and Hegde (2011) who reported significant variation for growth habit, seed shape, seed testa texture, seed coat colour and flower colour among chickpea germplasm. Stoilova and Pereira (2013) reported presence of wide variation for qualitative traits among cowpea genotypes. Theoretically, qualitative characters are controlled by a few genes with large effects and the expressions of these traits are more likely to be constant across environments and could be merely the results of genotypic differences. Genetic variation is the main interest of plant breeders and these variations can be utilized in future breeding programmes for improvement of chickpea.

The current results further indicated that seed yield $\mathrm{ha}^{-1}$ was positively and significantly correlated with plant canopy width, number of secondary branches plant ${ }^{-1}$, pods plant $^{-1}$ and biomass yield ha ${ }^{-1}$ but non-significantly correlated with other traits like number of primary branches plant ${ }^{-1}$, plant height and 100 seed weight (Table 5). The results therefore implied that increased seed yield ha ${ }^{-1}$ was not due to high number of primary branches plant $^{-1}$, tall plant height and high 100 seed weight but it was associated with high number of secondary branches plant $^{-1}$, larger plant canopy width, increased biomass yield ha ${ }^{-1}$ and increased number of pods plant ${ }^{-1}$. It evidently indicated that the positive and significant correlation among agronomic traits with seed yield played an important role in the increment of ultimate seed yield. Thus the aforementioned characters could be selected and improved simultaneously to boost yield and further improvement of chickpea in the region. Qureshi et al. (2004) recommended that characters with positive and significant correlation with yield can be utilized in hybridization of chickpea for the development of varieties in combination of all those characters with seed yield.

In line with the present results, previous studies in chickpea, have reported positive and significant correlation of seed yield with the number of secondary branches plant ${ }^{-1}$, number of pods plant ${ }^{-1}$ and biomass yield (Padmavathi et al., 2013), seed yield with biomass yield and harvest index (Ahmad et al., 2012). Other studies in chickpea, also reported negative and non-significant correlation of seed yield with plant height (Ali et al., 2011). Seed yield was negatively and significantly correlated with plant height and number of branches plant ${ }^{-1}$ (Yucel and Anlarsal, 2010). However, these results contradict to those previous findings by Vaghela et al. (2009) who reported positive and significant correlation of seed yield ha $^{-1}$ with 100 seed weight in chickpea. Non-significant correlation of seed yield with days to $50 \%$ flowering could be due the fact that early flowering genotypes give low yield than late growth. Generally earliness trait is at the expense of ultimate seed yield. Though earliness usually related with low yield, in circumstances of low rainfall areas, it enables the plants to use the available soil moisture and escape from biotic and abiotic stresses that occur late in the growing season.

Biomass yield $\mathrm{ha}^{-1}$ correlated positively and significantly with days to $50 \%$ flowering, days to $50 \%$ podding, plant canopy width, plant height, number of primary branches plant ${ }^{-1}$, number of secondary branches plant ${ }^{-1}$, days to $75 \%$ maturity and number of pods plant ${ }^{-1}$ (Table 5). A positive and significant correlation between seed yield $\mathrm{ha}^{-1}$ and biomass yield ha ${ }^{-1}$ indicated that both economic characters could be improved simultaneously. In some situations, wherever chickpea is produced as forage for livestock feed, it could be good opportunity for selection of genotypes with higher biomass yield $\mathrm{ha}^{-1}$. Larger plant canopy width, tall plant height, more number of primary and secondary branches plant ${ }^{-1}$, high number of pods plant ${ }^{-1}$ and late maturity could be associated with high biomass yield ha ${ }^{-1}$ and these characters could be improved concurrently to enhance the overall biomass yield and hence contributed to final seed yield production.

Number of pods plant ${ }^{-1}$ correlated positively and highly significant with days to $50 \%$ flowering, days to $50 \%$ podding, plant canopy width, plant height, number of primary and secondary branches plant ${ }^{-1}$ and days to $75 \%$ 
maturity while negatively and significantly correlated with pod filling period. These evidently indicated that late flowering, late podding, more number of primary and secondary branches plant ${ }^{-1}$, larger plant canopy width, tall plant height and late maturity could be contributed for more number of pods plant ${ }^{-1}$ and seed yield. In addition, negative and non-significant correlation of pods plant ${ }^{-1}$ with 100 seed weight indicated genotypes with more number of pods plant ${ }^{-1}$ could set small seeds due to competition for moisture and nutrients and vice versa.

There was positive and highly significant correlation of days to maturity with days to flowering, days to podding, plant canopy width, plant height, number of primary and secondary branches plant ${ }^{-1}$ (Table 5). These showed early maturing genotypes were not only early flowering and podding but they had small plant canopy width, short plant height, low number of primary and secondary branches plant ${ }^{-1}$ and vice versa. Similarly Malik et al. (2014) reported positive and significant correlation of days to maturity with number of primary branches plant ${ }^{-1}$, number of secondary branches plant ${ }^{-1}$ and number of pods plant ${ }^{-1}$.

The results of the principal components analysis showed that the first three principal components with eigenvalue greater than one explained $80.14 \%$ of the total variation among genotypes. The first principal component was associated with days to $50 \%$ flowering, days to $50 \%$ podding, plant canopy width, plant height, number of primary and secondary branches plant ${ }^{-1}$, days to $75 \%$ maturity, number of pods plant ${ }^{-1}$ and biomass yield ha ${ }^{-1}$ as positive contributors. The first principal component accounted for the highest variability in the data compared to succeeding components. The second principal component was positively related with number of leaflet leaf ${ }^{1}$ at podding, plant canopy width, plant height, pod filling period, number of pods plant ${ }^{-1}$, biomass yield ha ${ }^{-1}$ and seed yield ha ${ }^{-1}$. The results indicated that potential candidate traits for breeding materials could be obtained from genotypes in first and second principal components. Malik et al. (2014) reported that the first principal component was positively contributed through seed yield, biomass yield, number of pods plant ${ }^{-1}$, number of secondary branches plant ${ }^{-1}$ and plant height while days to flowering, days to maturity and 100 -seed weight were positively associated with $2^{\text {nd }}$ principal component in chickpea. Muniraja et al. (2011) also reported the first principal component was associated with number of pods plant ${ }^{-1}$, seed yield, plant height and number of secondary branches plant ${ }^{-1}$ while the second principal component correlated with days to flowering and days to maturity in chickpea germplasm. In cereal, Habibpour et al. (2012) reported the first five principal components explained $69.3 \%$ of the total variations where the $1^{\text {st }}$ principal component was linked with tiller numbers, fertile tillers, biological yield and grain yield in wheat.

\section{Conclusion}

It can be concluded from the above results that there is considerable genetic variation among genotypes for all studied characters. The ranking of genotypes for yield performance varied across environments. These could be attributed to a crossover type of genotype by environment interactions. High yielding genotypes across environments namely ICCV 05315, ICC 13461, ICCV 07313, ICC 13764 and ICCV 00302, were identified.

Seed yield $\mathrm{ha}^{-1}$ was positively and significantly $(\mathrm{p}<0.05)$ correlated with biomass yield ha ${ }^{-1}$, number of pods plant $^{-1}$, plant canopy width and number of secondary branches plant ${ }^{-1}$. This indicated the aforementioned traits were highly associated with seed yield and could be used for indirect selection of high seed yielding genotypes.

The results further showed that $80 \%$ of the total variation was explained by the first three principal components. The first principal component was associated with the main agronomic and yield related traits as positive contributors. Overall, the existence of substantial differences for agro-morphological and yield related traits, positive and significant association among agronomic traits with seed yield among the genotypes in this study, shows that the studied germplasm can be exploited for future breeding programmes of chickpea in the region.

\section{Acknowledgements}

The authors are grateful to African Development Bank (AFDB) through National Board of Higher Education of Eritrea (NBHE) for financial support of the study. The first author acknowledged Hamelmalo Agricultural College (HAC), Eritrea for granting him study leave to pursue higher education.

\section{References}

Ahmad, Z., Mumtaz, A. S., Nisar, M., \& Khan, N. (2012). Diversity Analysis of Chickpea (Cicer arietinum L.) Germplasm and its Implications for Conservation and Crop Breeding. Agricultural Sciences, 3(5), 723-731. http://dx.doi.org/10.4236/as.2012.35087

Ali, Q., \& Ahsan, M. (2012). Estimation of Genetic Variability and Correlation Analysis for Quantitative Traits in Chickpea (Cicer arietinum L.). International Journal for Agro Veterinary and Medical Sciences, 6(4), 241-249. http://dx.doi.org/10.5455/ijavms.20110601090444 
Ali, Q., Tahir, M. H., Sadaqat, S. A., Farooq, J., Ahsan, M., Waseem, M., \& Iqbal, A. (2011). Genetic Variability and Correlation Analysis for Quantitative Traits in Chickpea Genotypes (Cicer arietinum L.). Journal of Bacteriology Research, 3(1), 6-9.

Aslamshad, M., Pervez, H., Zafar, Z., Zia-Ul-Haq, M., \& Nawz, H. (2009). Evaluation of Biochemical Composition and Physiochemical Parameters of from Seeds of Desi Chickpea Varieties Cultivation in Arid Zones of Pakistan. Pakistan Journal of Botany, 41(2), 655-662.

Atta, B. M., Haq, M. A., \& Shah, T. M. (2008). Variation and Inter-Relationships of Quantitative Traits in Chickpea (Cicer arietinum L.). Pakistan Journal of Botany, 40(2), 637-647.

Bakhsh, A., Wahid, M. A., Bugt, R. A., Zahid, M. A., \& Ali, S. (2003). Evaluation of Chickpea Germplasm for Semi-Arid Zones of Baluchistan. International Journal of Agriculture and Biology, 5(2), 113-116.

Food and Agricultural Organization of the United Nations (FASOSTAT). (2012). Retrieved from http://faostat3.org/home/index/html

Gul, R., Khan, H., Bibi, M. Q., Ain, U., \& Imran, B. (2013). Genetic Analysis and Interrelationship of Yield Attributing of Traits in Chickpea (Cicer arietinum L.). The Journal of Animal and Plant Sciences, 23(2), 521-526.

Habibpour, M., Ahmadizadeh, M., \& Shahbazi, H. (2012). Assessment Relationship between Agro-morphological Traits and Grain Yield in Bread Wheat Genotypes under Drought Stress Condition. African Journal of Biotechnology, 11(35), 8698-8704. http://dx.doi.org/10.5897/AJB11.3421

Hegde, V. S. (2011). Morphology and Genetics of a New Found Determinate Genotype in Chickpea. Euphytica, 182, 35-42. http://dx.doi.org/10.1007/s10681-011-0447-5

IBPGR, ICRISAT, ICARDA. (1993). Descriptors for Chickpea (Cicer arietinum L.). International Board of Plant Genetic Resources, Rome, Italy; International Crops Research Institute for Semi-Arid Tropics, Patancheru, India and International Center for Agricultural Research in the Dry Areas, Aleppo, Syria.

Jagadish, N., \& Jayalakshmi, V. (2014). Combining ability Studies for Drought Tolerance Attributes in Kabuli Chickpea (Cicer arietinum L.). Electronic Journal of Plant Breeding, 5(3), 435-441.

Kaluli, W., Mwangi, H. M., \& Sira, F. N. (2011). Sustainable Solid Waste Management Strategies in Juja, Kenya. Journal of Agricultural Sciences and Technology, 13(1), 79-90.

Karuku, G. N., Gachene, C. K. K., Karanja, N., Cornelis, W., Verplancke, H., \& Kironchi, G. (2012). Soil Hydraulic Properties of a Nitisol in Kabete. Tropical and Subtropical Agroecos, 15, 595-609.

Khan, R., Farhatullah, \& Khan, H. (2011). Dissection of Genetic Variability and Heritability Estimates of Chickpea Germplasm for various Morphological Markers and Quantitative Traits. Sarhad Journal of Agriculture, 27(1), 67-72.

Kwon, S. H., \& Torrie, J. A. (1964). Heritability and Inter-relationship among Traits of Soybean Populations. Crop Science, 4(1), 196-198. http://dx.doi.org/10.2135/cropsci1964.0011183X000400020023x

Malik, S. R., Bakhsh, A., Ahsan, M. A., Iqbal, U., \& Iqbal, S. M. (2009). Assessment of Genetic Variability and Interrelationship among some Agronomic Traits in Chickpea. International Journal of Agriculture and Biology, 12(1), 1814-9596.

Malik, S. R., Shabbir, G., Zubur, M., Iqball, S. M., \& Ali, A. (2014). Genetic Diversity Analysis of Morpho-genetic Traits in desi Chickpea (Cicer arietinum L.). International Journal of Agriculture and Biology, 16, 956-960.

Malik, S., Bakhsh, A., Asif, M. A., Iqbal, U., \& Iqbal, S. M. (2010). Assessment of Genetic Variability and Interrelationship among Some Agronomic Traits in Chickpea. International Journal of Agriculture and Biology, 12, 81-85.

Mallikarjuna, B. P. S., Upadhyaya, H. D., Kenchana, G. P. V., Kullaiswamya, B. Y., \& Sing, S. (2003). Phenotypic Variation for Agronomic Characteristics in a Groundnut Core Collection for Asia. Field Crops Research, 84, 359-371. http://dx.doi.org/10.1016/S0378-4290(03)00102-3

Millan, T., Clarke, H. J., Siddique, K. H. M., Buhariwalla, H. K, Gaur, P. M., Kumar, J., ... Winter, P. (2006). Chickpea Molecular Breeding: New Tools and Concepts. Euphytica, 147, 81-103. http://dx.doi.org/10.1007/s10681-006-4261-4.

Mulwa, R. M. S., Kirmurto, P. K., \& Towett, B. K. (2010). Evaluation and Selection of Drought and Pod borer 
Tolerant Chickpea Genotypes for Introduction in Semi-arid Areas of Kenya. Second RUFORUM Biennial Meeting, September 20-24, 2010, Entebbe, Uganda.

Muniraja, C., Satish, R. G., Raju, C., \& Hart, M. (2011). Principal Component Analysis among Genotypes of Chickpea (Cicer arietinum L.). International Journal of Agricultural Sciences, 7(2), 382-386.

Oladejo, A. S., Akinwale, R. O., \& Obisesan, I. O. (2011). Interrelationships between Grain Yield and other Physiological Traits of Cowpea Cultivars. African Crop Science Journal, 19(3), 189-200.

Onyari, A. N., Ouma, J. P., \& Kibe, A. M. (2010). Effects of Tillage Method and Sowing Time on Phenology and Yield of Chickpea. Journal of Applied Biosciences, 34, 2156-2165.

Padmavathi, P. V., Murthy, S. S., Rao, V. S., \& Ahamed, L. M. (2013). Correlation and Path Coefficient Analysis in Kabuli Chickpea (Cicer arietinum L). International Journal of Applied Biology and Phyrmaceutical Technology, 4(3), 107-110.

Parameshwarapp, S. G., Salimath, P. M., Upadhyaya, H. D., Patil, S. S., \& Kajjidoni, S. T. (2012). Genetic Variability Studies in Minicore Collection of Chickpea (Cicer arietinum L.) under different Environments. Kamatak Journal of Agricultural Science, 25(3), 305-308.

Qureshi, A. S., Shaukat, A., Bakhsh, A., Arshad, M., \& Ghafoor, A. (2004). Assessment of Variability for Economically Important Traits in Chickpea (Cicer arietinum L.). Pakistan Journal of Botany, 36(4), 779-785.

Ramanappa, T. M., Chandrashekara, K., \& Nuthan, D. (2013). Analysis of Variability for Economically Important Traits in Chickpea (Cicer arietinum L.). International Journal of Research in Applied, Natural and Social Sciences, 1(3), 133-140.

Roy, S., Islam, M. A., Sarker, A., Malek, M. A., Rafii, M. Y., \& Ismail, M. R. (2013). Determination of Genetic Diversity in Lentil Germplasm based on Quantitative Traits. Australian Journal of Crop Science, 7(1), 14-21.

SAS Institute Inc. (2013). SAS® Enterprise Miner ${ }^{\mathrm{TM}}$ 12.3: Administration and Configuration (2nd ed). Cary, NC: SAS Institute Inc.

Sombroek, W. G., Braun, H. M. H., \& Vanderpouw, B. J. A. (1982). Exploratory soil survey. Report No. E1. Ministry of Agriculture, Nairobi Kenya.

Stoilova, T., \& Pereira, G. (2013). Assessment of the Genetic Diversity in a Germplasm Collection of Cowpea (Vigna unguiculata (L.)Walp.) using Morphological Traits. African Journal of Agricultural Research, 8(2), 208-218. http://dx.doi.org/10.5897/AJAR12.1633

Tesfamichael, S. M., Githiri, S. M., Nyende, A. B., Rao, G. N. V. P. R., Odeny, D. A., Rathore, A., \& Kumar, A. (2014). Assessment of Genetic Variation and Heritability of Agronomic Traits in Chickpea (Cicer arietinum L.). International Journal of Agronomy and Agricultural Research, 5(4), 76-88.

Upadhyaya, H. D., Gowda, G. L. L., \& Sastry, D. V. S. S. R. (2008). Management of Germplasm Collections and Enhancing their Use by Mini Core and Molecular Approaches (pp. 35-70). Workshop Capacity Building for risk Management Systems, Genet Resources.

Upadhyaya, H. D., Salimath, P. E., Gowda, C. L. L., \& Singh, E. S. (2007). New early-maturing Germplasm lines for Utilization in Chickpea Improvement. Euphytica, 157, 195-208. http://dx.doi.org/10.1007/s10681-007-9411-9

Upadhyaya, H. D., Ortiz, R., Bramel, P. J., \& Singh, S. (2002). Phenotypic Diversity for Morphological and Agronomic Traits in Chickpea Core Collection. Euphytica, 123, $333-342$. http://dx.doi.org/10.1023/A:1015088417487

Vaghela, M. D., Poshiya, V. K., Savaliya, J. J., Davada, B. K., \& Mungra, K. D. (2009). Studies on characters association and path analysis for seed yield and its components in chickpea (Cicer arietinum L.). Legume Resources, 32(4), 245-249.

Yimram, T., Somta, P., \& Srinives, P. (2009). Genetic Variation in Cultivated Mungbean Germplasm and its Implication in Breeding for High Yield. Field Crops Research, 112, 260-266. http://dx.doi.org/10.1016/j.fcr.2009.03.013

Yucel, D. O., \& Anlarsal, A. E. (2010). Determination of Selection Criteria with Path coefficient Analysis in Chickpea (Cicer arietinum L.) Breeding. Bulgarian Journal of Agricultural Science, 16(1), 42-48. 
Zelalem, Z. (2014). Evaluation of Agronomic Traits of Different Haricot Bean (Phaseolus Vulgaris L.) Lines in Metekel Zone, North Western part of Ethiopia. Wudpecker Journals, 3(1), 39-43.

\section{Copyrights}

Copyright for this article is retained by the author(s), with first publication rights granted to the journal.

This is an open-access article distributed under the terms and conditions of the Creative Commons Attribution license (http://creativecommons.org/licenses/by/3.0/). 